\title{
Las restricciones del conocimiento notacional
}

\author{
Liliana TOLCHINSKY y ANNETte KaRmilofF-Smith \\ Universidad de Barcelona y MCR de Londres
}

\begin{abstract}
Resumen
Sólo los seres bumanos utilizan berramientas para dejar trazas permanentes de sus actos comunicati. vos. Esta capacidad notacional se manifiesta en distintos sistemas notacionales: dibujo, escritura o notación numérica que se distinguen entre sí por sus elementos, por sus regularidades combinatorias y por cumplir distintas funciones. En este trabajo exploramos cómo comprenden los niños entre 3:8 y 6:6 años de edad las relaciones entre sistemas utilizando dos situaciones de producción de notaciones. En la primera los niños debian escribir qué $y$ cuántos objetos aparecen dibujados en una serie de tarietas. El objeto de esta situación fue verificar sus posibilidades de producir notaciones diferenciadas se. gún las restricciones formales y adecuadas a las funciones especificas de cada sistema. En la segunda, los niños debian inventar letras, números y palabras que no existen. El propósito fue averiguar en qué medida las restricciones formales $y$ la adecuación funcional que bubieran demostrado en la primera situación estaban implicitas en sus procedimientos de producción o podian ser intemamente explicitadas, y por tanto transgredidas. Los resultados demuestran que desde muy temprana edad los niños producen notaciones diferenciadas según las restricciones formales propias de la escritura y del sistema de notación numérico. Sin embargo, la diferenciación formal no asegura el uso adecuado de las notaciones para sus funciones específicas. A pesar del conocimiento de las restricciones formales de cada sistema, algunos niños recurren al dibujo para indicar qué objetos hay en una figura o a la escritura para indicar cuántos objetos hay. Sólo cuando el conocimiento de las restricciones formales que estaba:implícito en los procedimientos de producción se explicita internamente, y puede por tanto ser transgredido, el niño utiliza cada sistema según su función específica.
\end{abstract}

Palabras clave: Conocimiento notacional; Escritura; Notación numérica; Diferenciación formal; Adecuación funcional.

\section{Restrictions of Notational Knowledge}

\section{Abstract}

Only bumans use tools in order to leave traces of their communicative acts. This capacity can be expressed in different notational systems: drawing, writing, numbers. Eacb system includes different elements, is characterized by different combinatorial regularities, and fulfils a distinct communicative function. The study explores children's understanding of the relationship between systems. Two production tasks were designed. In the first task, children were asked to write what and how many objects appeared on a series of pictures. The purpose was to verify the extent to which children's production appeared differentiated according to the constraints of each notational system. In the second task, they were asked to invent numbers, letters, and words that do not exist. The aim of this task was to discover the extent to which the constraints used in the production task were implicitly represented in the structure of the production procedures or were explicitly represented and therefore available for purposeful manipulation. Our results show that children as young as $4 \mathrm{yrs}$. produced differentiated notations. Notations were constrained by the formal limitations of each notational system. However, formal differentiation did not assure the functional adequacy of notations. Some children resorted to drawing in order to show which objects appeared in a picture or to writing to indicate how many objects there were in the picture. Only after the children's constraints, implicit in the children's production procedures, became explicitly represented and available for intermal manipulation could the child use each system according to its specific function.

Key words: Notational knowledge; Writing; Number notation; Formal differentiation; Functional adequacy.

Correspondencia con las autoras: La correspondencia puede ser dirigida a cualquiera de las dos autoras. Liliana Tolchinsky Landsmann, Instituto de Ciencias de la Educación, Calle de los Angeles, 18, 08001 Barcelona, España/Annette Karmiloff-Smith, MRC Cognitive Development Unit, 17 Gordon St., WCI OAH, U.K.

Original recibido: septiembre, 1991. Revisión recibida: enero, 1993. Aceptado: febrero, 1993.

(C) 1993 by Aprendizaje, ISNN 0210-3702

Infancia y Aprendizaje, 1993, 62-63, 19-51 
Numerosas metáforas se han utilizado para caracterizar el conocimiento que los niños poseen de un dominio específico. Se habla de los niños como linguistas, para indicar el conocimiento que tienen acerca de la lengua; de los niños como psicólogos, para referirse a las ideas que tienen acerca de los estados intencionales propios o ajenos. En este trabajo nos ocupamos del conocimiento que el niño tiene del dominio notacional: el dominio de las formas gráficas que se usan para registrar y transmitir información. Por ejemplo, cifras, notación musical, notación química, escritura, etc. Podríamos decir, metafóricamente, que nos ocupamos del niño como notador. En trabajos anteriores hemos distinguido entre lo notacional en sí, como dominio de conocimiento y lo notacional como instrumento de representación y comunicación (Tolchinsky Landsmann y Karmiloff Smith, 1992). Esta distinción sirvió para constatar que niños muy pequeños, antes de ser alfabetizados, son sensibles a lo notacional aun cuando no sepan utilizar las notaciones como instrumento de representación y comunicación. En este trabajo volvemos sobre esta distinción e intentamos explicar qué suponemos tiene que suceder en la mente del niño para que pase de una a otra forma de conocimiento.

\section{¿QUE ES LA CAPACIDAD NOTACIONAL?}

Es la capacidad de utilizar herramientas para dejar marcas permanentes de actos intencionales, una capacidad que sólo el ser humano posee (Karmiloff Smith, 1990). Otras especies, no humanas, como los moluscos o insectos dejan trazas de sus desplazamientos espaciales que les permiten volver al punto de partida, pero para ello utilizan sus propios excrementos, sin que entren en juego, por tanto, herramientas externas al propio cuerpo. Esas marcas espaciales, además, son claramente no intencionales y se realizan in situ, no para simbolizar los desplazamientos. Ni siquiera entre los chimpancés, que han demostrado capacidades comunicativas y representacionales, pudo observarse la producción de marcas gráficas con función mnemónica (Premack, 1986; Boysen y Berntson, 1989; Boysen, comunicación personal).

Los desarrollos más recientes en paleontología confirman que tampoco entre nuestros antepasados más cercanos existió el registro intencional. El Homo erectus, los neandertales y otros, poseían capacidades mentales de orden elevado, como lo indica la gama de útiles y otros artefactos que utilizaban. Pero sólo el Homo sapiens posee el tipo de razonamiento abstracto - que incluye los usos numéricos y estéticos - que indentificamos como específicamente humano (Gould, 1991: 329). Las diferencias entre las capacidades del Homo sapiens y de otras especies más o menos emparentadas no radican en la posibilidad de computar o de usar herramientas para resolver problemas sino en el registro intencional del cómputo y en la utilización de herramientas para registrar.

Explorar la capacidad notacional del niño es, por tanto, explorar su capacidad para interpretar y producir marcas gráficas que se utilizan intencionalmente para registrar promesas, ritmos, cantidades, creencias, etc. Esta capacidad ha dado origen, históricamente, a diferentes notaciones de las cuales las más difundidas en la cultura occidental son el alfabeto y las cifras arábigas. 


\section{¿QUE SON LOS SISTEMAS NOTACIONALES?}

Las marcas producidas intencionalmente pueden ser icónicas o no icónicas. La pintura y el grabado son habitualmente icónicos, mientras que el alfabeto o las cifras arábigas no lo son. Existe un cierto desacuerdo en la literatura respecto a las características que definen lo icónico y a las condiciones que deben cumplirse para que marcas gráficas constituyan una notación. (Dilucidar esta polémica va más allá de las posibilidades de este trabajo pero véase Willats, 1977; Freeman, 1987.)

Goodman (1968) y Harris (1992), por ejemplo, coinciden en que para hablar de notación deben darse las siguientes condiciones: 1) un conjunto de objetos o de unidades abstractas, 2) en número limitado y de forma definida y 3) formando compuestos de acuerdo con restricciones definidas. Las «unidades abstractas», algo equivalente al constructo «fonema» en lengua oral, permiten reconocer a los usuarios del sistema que se trata de la letra «a» aunque aparezca escrita por personas de muy diverso estilo caligráfico. Es decir, permite identificar la unidad a pesar de las variaciones individuales. Goodman denomina a las «unidades abstractas» caracteres, y a las producciones individuales concretas, inscripciones. En este trabajo utilizamos esta terminología, además del término «elemento», para referirnos a las unidades de las distintas inscripciones.

Más allá de esta coincidencia, no hay acuerdo respecto a si las unidades tienen significado y regularidades (de orden o jerarquía) en sí, fuera de los sistemas que las utilizan. Harris sostiene que sí, que en la notación se establece siempre una relación de equivalencia o una relación de prioridad entre dos unidades. Según Harris, entre las letras del alfabeto hay un orden (alfabético) que no depende del sistema de escritura y entre las cifras hay otro orden -el 1 anterior en el orden al 2- que tampoco depende de que éstas se usen como notación de cardinalidades. El mismo autor sostiene, en cambio, que las relaciones de referencia (o semántica propia de los elementos notacionales) son función de los sistemas que utilizan esas notaciones. El alfabeto tiene una referencia diferente cuando se utiliza como notación en el álgebra, como notación numérica en el sistema Romano o cuando se usa como sistema de escritura.

Parecería que esta discusión gira en torno a la distinción que hemos hecho entre lo notacional en sí y como instrumento de representación y comunicación. Esta distinción está clara para los matemáticos, que no confunden entre la notación y los números que ella puede representar. A los no matemáticos, sin embargo, nos resulta fácil identificar una notación con su empleo más corriente. Estamos tentados de suponer, por ejemplo, que la cifra 3 tiene siempre el mismo valor numérico, porque la utilizamos sobre todo para contar, para expresar una cierta idea cuantitativa. Sin embargo, la cifra 3 se mantiene idéntica a sí misma cuando sirve como prefijo telefónico y significa Barcelona.

En este trabajo nos concentramos en dos sistemas notacionales: la escritura alfabética, un sistema que utiliza como notación el alfabeto Latino, y la notación numérica decimal, un sistema que utiliza las cifras arábigas. Las relaciones de referencias que definimos responden al valor de los caracteres - las letras del alfabeto y las cifras - en esos sistemas. Cuando el alfabeto se usa en un sistema de escritura, sus caracteres refieren a segmentos fonológico de rango definido consonánticos y vocálicos. Cuando las cifras se usan en un sistema de notación decimal refieren a orden y cardinalidad en base diez.

La distinción hecha entre notacional en sí y como instrumento de representa- 
ción y comunicación nos servirá para entender mejor los distinto aspectos involucrados en el conocimiento notacional. Podremos distinguir entre un niño que conoce las letras por su nombre, su forma y su orden y no conoce su referencia o niños que conocen todo lo anterior y las relaciones de referencia (usan letras con valor sonoro convencional), pero utilizan la escritura para representar cantidad. Por ejemplo, repite tres veces la escritura del nombre de un objeto porque éste aparece dibujado tres veces. La misma distinción sirve para interpretar los distintos aspectos involucrados en el conocimiento de los compuestos. Cuando se utilizan en textos reales, los compuestos de escritura suelen tener muy pocas letras consecutivas repetidas (una serie de letras repetidas no es una palabra) y el número de letras por compuesto suele darse en un rango bastante restringido. Además, las letras, en nuestro sistema de escritura, pueden escribirse ligadas o separadas. Estas restricciones son diferentes para los compuestos de cifras. Pueden encontrarse compuestos que incluyen la misma cifra muchas veces (una serie de cifras repetidas sigue siendo un número), números «notados» por una sola cifra o por muchísimas. Además, las cifras no se escriben ligadas.

Resumiendo: el tipo de elementos, el número de elementos por compuesto, la repetición y la ligazón son restricciones que diferencian la escritura del sistema numérico. Si volvemos a la distinción hecha anteriormente podemos suponer que haya niños que diferencien entre los elementos y las restricciones que rigen los compuestos y no entre las relaciones de referencia de cada sistema. Podemos también suponer que haya niños que conocen todo lo anterior y no utilizan los sistemas adecuadamente. En este trabajo nos propusimos evaluar estos distintos aspectos del conocimiento en una situación de producción de notaciones. Estudiamos las relaciones entre sistemas desde el punto de vista del sujeto que los está aprendiendo. Antes de presentar las situaciones usadas revisaremos algunas de las explicaciones históricas y ontogenéticas que se han dado acerca de las relaciones entre sistemas.

\section{¿COMO SE ORIGINARON, HISTORICA Y ONTOGENETICAMENTE, LAS DIFERENCIAS ENTRE SISTEMAS NOTACIONALES?}

Una de las explicaciones históricas más habituales es la de la continuidad entre las expresiones icónicas y las no icónicas. A menudo se sostiene que en la base de los sistemas de escritura está el dibujo (por ejemplo, Gelb, 1963: 27). Aunque esta suposición suena razonable, su validez ha sido puesta en duda por tres series de hechos:

1) Desde las primeras apariciones de notaciones, en el período paleolítico superior, entre -29.000 y -11.000 años, tanto en Asia occidental como en Europa, las formas icónicas, que suelen representar animales, coexisten con las no icónicas, que aparecen en forma de trazos paralelos marcados en huesos. La función de estas notaciones, tanto la de las icónicas como la de las geométricas, sólo puede ser hipotetizada. Marschak (1972), por ejemplo, considera que los huesos tallados son calendarios lunares en los cuales cada marca registra la aparición de una luna; y Leroi Gourhan (1971) piensa que los animales dibujados simbolizan una compleja cosmología. Lo importante, sin embargo, es que ya desde el inicio del desarrollo histórico de la notación, las dos categorías están presentes. La forma icónica no es, por tanto, la más primitiva.

2) Se ha establecido una clara relación entre la escritura y una notación muy 
primitiva, utilizada aparentemente con fines contables. Se trata de unos bastones de arcilla en los cuales aparecen dos clases de marcas. Unas, agrupadas y repetidas que funcionaban como control - por correspondencia- de la cantidad de objetos transportados o trocados. Otras distintas que supuestamente indicaban qué objeto era el transportado (por ejemplo, ovejas). Estas marcas funcionaban como clasificadores de las marcas repetidas. Es decir, para separar entre aquellas marcas que correspondían a ovejas de aquellas que correspondían a cerdos. Los clasificadores no se repetían, uno bastaba para indicar el animal u objeto. De los clasificadores surge aparentemente la escritura (Harris, 1990; Schmandt-Besserat, 1977, 1978, 1990).

3) Los más simples antecesores de la escritura contienen elementos no pictóricos. Por ejemplo, los jeroglíficos poseían tres tipos diferentes de caracteres: ideogramas, fonogramas (consonantes) y determinativos. Los dos últimos indican, respectivamente, si el primero debe interpretarse en su sentido original o derivado y cuál es la función gramatical de la palabra.

Resulta claro, pues, que la escritura no deriva del dibujo en ningún sentido simple de derivación y que ciertas restricciones notacionales - como la repetición para corresponder cuantitativamente y la no repetición para la clasificación cualitativa- están presentes ya en formas notacionales muy primitivas.

En cuanto a la ontogénesis, la relación entre sistemas se ha explicado desde dos posiciones diferentes. Una de ellas sostiene la continuidad y el origen común de las formas de expresión simbólica y explica su evolución por mecanismos generales del desarrollo. La segunda sostiene que «la estructura mental es rica y variada», es decir, que es especializada ab initio. Denominamos a la primera semiótica y a la segunda modular. Los representantes más populares de la posición semiótica son Bruner, 1964; Piaget, 1946, y Vygotsky, 1978. Estos autores coinciden en el origen común y en la continuidad ontogenética, pero difieren en la importancia que le atribuyen a lo simbólico en el desarrollo. Vygotsky argumenta explícitamente en favor de una continuidad entre el gesto, el dibujo y escritura (Vygotsky, 1978). Piaget, en cambio, no habla de relaciones entre dibujo y la escritura o notación numérica pero coincide con Vygotsky, ya que para él todas las actividades simbólicas -lenguaje, imágenes mentales, gestos simbólicos, etc.derivan de una función semiótica general (Piaget, 1946). Las actividades simbólicas son instrumentos para - representar, comunicar- y emergen como culminación del período sensoriomotor, que carece de actividad simbólica. Las actividades sensomotoras, preverbales, dan origen a las actividades verbales y la función de las restricciones propias de cada una de las actividades simbólicas es secundaria, ya que sólo ofrece diferente resistencia a los procesos generales de asimilación y acomodación. Piaget defiende, además, la primacía de lo conceptual sobre lo simbólico. Son los estadios del desarrollo cognitivo los que determinan «la naturaleza y la forma» (Ferreiro y Sinclair, 1971) de las estructuras lingüísticas y, por extensión, de cualquier actividad simbólica. Lo simbólico, «los aspectos figurativos del conocimiento», dependen de los aspectos operatorios en las dos acepciones de la palabra dependencia: subordinación y derivación.

Desde esta perspectiva la escritura y la notación numérica serían una expresión más elevada pero continua de la función semiótica. Los procesos de adquisición de cada uno de los sistemas serían similares y se explicarían por los procesos generales del desarrollo. Se postula una neutralidad de dominio. Las formas sim- 
bólicas son concebidas como instrumentales, así, para poder llegar a comprenderlas habrá que utilizarlas. La competencia notacional dependería del desarrollo nocional.

Algunos datos provenientes de la neuropsicología resultan difíciles de explicar desde una posición de neutralidad de dominio y continuidad. En especial los casos de disfunción selectiva en los cuales, por ejemplo, adultos después de una lesión cerebral no reconocen la letra $\mathrm{H}$ pero sí el dibujo de una escalera que tiene forma muy similar; $\mathrm{u}$ otros no pueden escribir palabras pero sí números o dibujos (Shallice, 1988, Gardner, 1974; Ellis y Young, 1991); casos de niños hidroencefálicos o con síndrome de Williams cuyo lenguaje presenta una organización sintáctica y semántica adecuada a pesar de su enorme retraso intelectual (véase Curtiss y Yamada, 1981; Cromer, 1988, y la discusión en Karmiloff Smith, 1990). Otros datos provenientes de trabajos en psicología cognitiva demuestran que la heurística del desarrollo sensoriomotor no puede explicar el desarrollo linguístico. En un estudio longitudinal con niños sordos profundos, Petitto (1987) demuestra que aunque los niños utilizan el gesto de autorreferencia (señalarse a sí mismo) antes de adquirir cualquier forma de lenguaje de signos, cuando comienzan a «hablar» en lenguaje de signos, la adquisición de los pronombres de primera y segunda persona sigue un proceso similar al que se da en los niños oyentes, incluso con producción de errores. No se detecta, pues, una continuidad entre el gesto, que los niños ya poseían (formas preverbales) y la expresión de la autorrefencia en el Sistema Americano de Signos ASL (formas verbales). Estas evidencias ponen en duda tanto la continuidad como la neutralidad de dominio de los sistemas simbólicos.

Desde otro campo distinto, el de la enseñanza de las matemáticas, se cuestiona también la dependencia de lo notacional a lo nocional y se defiende la necesidad de considerar, separadamente, la peculiaridad del conocimiento notacional. Es evidente que no existe una traducción directa de la comprensión de determinado concepto a su notación. Muchas veces la posibilidad de representar gráficamente una operación o una relación facilita su comprensión. Es probable que ciertos desarrollos matemáticos no hubieran sido posibles sin la existencia de un sistema notacional que los precediera. La necesidad y las exigencias que plantea el uso notacional en las matemáticas es tan extrema que Kaput (1987) propone la necesidad de elaborar una teoría de lo notacional.

Teniendo en cuenta todo lo anterior y en contraste con la perspectiva semiótica, la perspectiva «modular» postula una especificidad de dominio. La noción de especifidad se ha utilizado con acepciones muy diversas (véase Tolchinsky, Landsmann y Teberosky, 1993). Por ejemplo, para oponerse a posturas que defienden la existencia de habilidades cognitivas universales válidas para resolver problemas en cualquier contexto y demostrar que hay individuos y grupos sociales que pueden ser sumamente hábiles en determinados contextos y muy poco hábiles en otros. Esta acepción de «especificidad» es diferente de la que postula Fodor, por tomar otro ejemplo. Fodor (1983) sostiene que la mente está formada por módulos de propósitos específicos, es decir, que computan estrictamente los estímulos para los cuales están genéticamente preparados. No importa lo caótico de la información ambiental, cada clase de estímulo - perceptual, lingüístico, numeral, etc.- irá al correspondiente módulo.

En otros trabajos una de nosotras (cf. Karmiloff-Smith, 1986, 1992) ha propuesto detalladamente un modelo de desarrollo humano que incluye una consi- 
deración de las predisposiciones innatas del individuo y una concepción epigenética constructivista del aprendizaje. Sólo de esta manera parece posible proporcionar un modelo explicativo tanto del despegue inicial como de la flexibilidad y capacidad creativa inherentes al ser humano. En este modelo, sobre el cual volveremos en las próximas páginas, el desarrollo del ser humano presenta dos tipos de cambios complementarios: una progresiva modularización hacia estructuras que funcionan de forma automática y especializada pero menos accesible y un progresivo acceso a la información, a partir de procedimientos específicos cuyos componentes se van volviendo cada vez más explícitos y más accesibles.

Consideramos que la escritura y la notación numérica constituyen dominios diferenciados y suponemos que requieren procesos de aprendizaje especializados. En otros trabajos mostramos que los procedimientos clasificatorios de los niños muestran que éstos son sensibles a esta diferencia, mucho antes de ser alfabetizados (Tolchinsky, Landsmann y Karmiloff-Smith, 1992). En este trabajo completamos nuestra exploración de la diferenciación en una situación de producción. Supusimos que, en esta situación, los niños producirán diferentes elementos y diferentes regularidades en sus compuestos. Pero también supusimos que esta diferenciación no garantizaría el uso adecuado de la escritura y de la notación numérica como instrumentos de representación y comunicación. Intentamos, pues, acceder a los procesos intrapsicológicos que suponemos facilitan el paso de la diferenciación al uso adecuado. La hipótesis fue que para alcanzar este último nivel de diferenciación, el niño debía acceder a una explicitación de los aspectos notacionales (tipo de elementos, número de elementos en los compuestos, repetición, etc.) que le sirven para diferenciar notaciones a nivel procedural. No estamos diciendo con esto que debería poder explicarnos verbalmente por qué una notación $\mathrm{x}$ le parece buena para escribir y otra no, nos estamos refiriendo a una explicitación de nivel intermedio entre el nivel procedural y el nivel de explicación verbal explícita. Para entender estas afirmaciones debemos volver al modelo de cambio representacional que nos sirve de marco de referencia y desde el cual se explican estos niveles de explicitación. Este modelo fue útil para dar cuenta de aspectos léxico-morfológicos en la adquisición del lenguaje (Karmiloff-Smith, 1979, 1986a y 1986b); de distintas conductas en situaciones de resolución de problemas (Karmiloff-Smith, 1979, 1984), así como de las restricciones que se dan al cambio representacional (Karmiloff-Smith et al., 1990). Como este modelo ha sido desarrollado en detaIle (Karmiloff-Smith, 1986a, 1992a y 1992b) presentamos aquí solamente una breve reseña de los aspectos relevantes para este trabajo.

Pensamos que existen tres maneras básicas de adquirir conocimiento. Una, por especificación innata, a través de procesos evolutivos. El tiempo biológico y las condiciones ambientales permitirán su maduración. La segunda consiste en agregar nuevas representaciones por interacción activa con personas, objetos y modelos culturales. Una tercera e importante manera de ganar nuevo conocimiento es explotando el conocimiento ya representado.

La mayoría de las teorías psicológicas se inclinan por la primera o la segunda de las vías de adquisición del conocimiento. El modelo que estamos defendiendo prueba que ni la primera ni la segunda pueden por sí mismas, sin intervención de la tercera vía, explicar el cambio representacional. Esta tercera vía implica un proceso endógeno en el cual la mente explora conocimiento que ya ha almacenado - tanto en forma innata como por interacción-, re-represen- 


\section{6}

tando recursivamente sus propias representaciones internas. $\mathrm{O}$ de manera más precisa, representando iterativamente lo que sus representaciones internas representaron originalmente. El modelo supone por lo menos tres fases o niveles en las cuales tiene lugar la Redescripción Representacional. Es importante senalar que estos niveles son fases recursivas en distintos dominios o situaciones, no estadios de desarrollo.

En la primera fase el conocimiento inicial innato y aquel que se adquiere posteriormente se encuentran implícitamente representados. Está contenido en procedimientos que funcionan eficientemente y se activa en respuesta a estímulos ambientales, pero sus componentes no son aún accesibles a otras partes del sistema cognitivo. El conocimiento procedural es conocimiento que está en el sistema pero no para el sistema. Después de procesos de redescripción representacional, en un segundo nivel, el conocimiento está representado más explícitamente. Esto quiere decir que el conocimiento que era parte de los procedimientos que funcionaban eficientemente se vuelve accesible a otras partes del sistema cognitivo como estructura de datos. El conocimiento almacenado ya lo es para el sistema. No significa esto que el conocimiento sea conscientemente accesible o susceptible de ser verbalizado explícitamente, sino que el conocimiento es internamente accesible para su uso por otras partes del sistema. Este es el nivel de explicitación al que nos referimos antes como necesario para pasar de una diferenciación formal a una funcional. Después de otros procesos de Redescripción Representacional, en un nivel superior, el conocimiento puede verbalizarse y puede ser comunicado a otros. En otras palabras, elementos del mismo conocimiento son rerrepresentados mediante diferentes niveles de abstracción. Gran parte del desarrollo cognitivo consiste en re-describir internamente lo adquirido por interacción exógena; que ya funciona eficientemente.

En las ideas de Piaget acerca de los procesos de toma de conciencia encontramos importantes antecedentes de las ideas que estamos discutiendo (Piaget, 1976). Piaget habla de procedimientos exitosos que por procesos de interiorización se convierten en contenido del pensamiento. Piaget también insiste en lo graduado (y no dicotómico) de la toma de conciencia aunque no analiza los niveles intermedios entre el uso - los procedimientos- y la reflexión consciente, que generalmente identifica con la posibilidad de explicitación verbal. El modelo que estamos presentando rompe con la dicotomía conocimiento implícito versus conocimiento explícito, proponiendo niveles intermedios que explican mejor la complejidad del funcionamiento mental. Es importante resaltar que la Redescripción Representacional no está provocada por presiones «comunicacionales» ni por la inadaptación o fracaso de las fases anteriores. Ni en el desarrollo del lenguaje, ni en ninguno de los dominios estudiados - resolución de problemas físicos, dibujo, etc.- el sujeto pasa a niveles más abstractos de representación solamente para ser mejor entendido o porque fracasa en la resolución de problemas. A diferencia de la tan divulgada función del conflicto en el aprendizaje, provocada por las primeras ideas piagetianas, este modelo resalta la posibilidad de que el éxito provoque aprendizaje.

Veamos para ilustrar lo antedicho un estudio sobre el sistema de determinantes realizado con niños francófonos. Karmiloff-Smith (1979) demostró que estos niños adquieren muy temprano un dominio conductual de este subsistema. Los niños son, desde edad muy temprana, sensibles a los contextos en los cuales es necesario utilizar «un» como artículo indefinido (un) o «un» como nu- 
meral (uno). Lo hacen tan bien como cualquier adulto y por tanto son perfectamente comprendidos. Pero un par de años más tarde comienzan a cometer errores que indican que no pueden utilizar la misma palabra «un» con las dos funciones. Para contextos en los cuales la distinción debe ser marcada, utilizan «un $x »$ pero para indicar la referencia indefinida,utilizan en cambio un partitivo «un de x» (en aquellos contextos en los cuales un numeral es requerido). Esta marca redundante es luego abandonada y los niños vuelven a utilizar la misma forma superficial (un) para ambas funciones.

Si el cambio estuviera motivado sólo por razones de mejor comunicabilidad, de fracaso o inadaptación, no habría razón alguna para modificar lo que ya se estaba haciendo perfectamente bien. Lo que éste modelo sostiene es que, más allá del éxito conductual y de las presiones externas, existen razones endógenas para la reorganización representacional. La disminución en la eficiencia conductual, manifestada en el aumento de errores, resulta de una confrontación interna de aspectos que antes estaban separados. Los errores indican un trabajo interno que finalmente dará lugar a una relación sistemática entre aspectos que antes estaban yuxtapuestos.

Otro estudio en el dominio del dibujo, diseñado para tratar de comprender cuáles son las restricciones del cambio representacional (Karmiloff-Smith, 1990), demuestra la vigencia de la Hipótesis de RR en otro dominio. Todos los niños que participaron en el experimento -entre 5 y 9 años de edad - sabían dibujar rápida y eficazmente casas y figuras humanas. Pero eso no significa que los componentes del procedimiento - dibujar una casa - fueran accesibles como dato (interno), ya que podrían estar meramente implícitos en la secuencia del procedimiento. Para probar si efectivamente era así se les pidió que dibujaran una casa que no existe; un bombre que no existe. La idea era que para producir una casa que no existe el niño debía descomponer el procedimiento por el cual producía casas (que existen) en sus partes componentes y convertir esas partes en datos sobre los cuales trabajar internamente para producir las diferencias que darían como resultado casas que no existen. Los resultados muestran que los niños más pequeños, que saben dibujar eficazmente casas y hombres, no lograron, sin embargo, dibujar una casa que no existe. Es decir, no fueron capaces de producir ninguna modificación en las casas para que parecieran casas inexistentes. Los niños mayores, en cambio, lograron desarrollar una flexibilidad representacional creciente y pudieron dibujar casas u hombres que no existen. Por ejemplo, casa con alas u hombres con varias cabezas.

La Hipótesis de RR nos sirvió de base para establecer la hipótesis de la relación entre la posibilidad de acceder internamente a los aspectos formales del conocimiento notacional como condición para producir notaciones funcionalmente adecuadas y para diseñar algunas de las situaciones experimentales en el dominio de la escritura y la notación matemática.

\section{EL ESTUDIO EMPIRICO}

Numerosos estudios sobre el desarrollo de la escritura en diversas lenguas (Ferreiro y Teberosky, 1979; Frith, 1986; Tolchinsky, Landsmann y Levin, 1985, 1990) y sobre la notación numérica (Allardice, 1977; Hughes, 1986; Sinclair y Sinclair, 1984; Sastre y Moreno, 1976) han demostrado la precocidad del conocimiento infantil y las distintas fases por las cuales el niño pasa al intentar com- 
prender cada uno de los sistemas. El niño aprende, aparentemente, de la observación y exploración de las distintas expresiones notacionales que existen en su medio y al participar en las múltiples actividades en las que se usa la escritura y la notación numérica. Este conocimiento se desarrolla antes de que el niño escriba o use los números para comunicarse. Los estudios mencionados, sin embargo, han investigado cada uno de los sistemas separadamente y sabemos poco acerca de cómo percibe el niño las relaciones entre los sistemas. En este trabajo nos concentramos en la exploración de estas relaciones. Para ello nos planteamos las siguientes cuestiones:

$¿$ Los sistemas notacionales aparecen inicialmente diferenciados o se confunden con el dibujo? ¿La eventual diferenciación es un punto de partida, probable resultado de la experiencia de niños y niñas con el medio ambiente notacional? o ¿̇es el producto de la enseñanza formal?

¿Cómo diferencian los niños entre dibujo, escritura y notación numérica? ¿Por los elementos? ¿Por los tipos de compuestos que son posibles en uno u otro sistema? ¿O por sus relaciones de referencia? ¿ $\mathrm{O}$ por la función que cada uno cumple?

$¿ E l$ conocimiento de las diferencias formales (como dominio de conocimiento) precede o es una consecuencia del uso notacional (como instrumento)?

Presentamos los resultados de una de las situaciones experimentales con niños entre 4 y 6 años de edad que fue diseñada para responder a esas preguntas.

\section{Situación experimental I}

El propósito es lograr que los niños produzcan notaciones diferentes y poder analizar las diferencias. Supusimos que si les mostrábamos a los niños una serie de tarjetas con dibujos de los mismos objetos en distinta cantidad y les pedíamos que escribieran para saber qué objetos había dibujados y luego les pedíamos que «pusieran» para saber cuántos objetos había, conseguiríamos obtener una notación correspondiente a lo que el niño entendía por escritura y otra correspondiente a lo que el niño entendía por notación de la cantidad. Intentamos ser lo más explícitos posible tanto en cuanto al contenido de la instrucción como a su expresión linguística. Por esa razón utilizamos el verbo escribir cuando pretendíamos que representasen los objetos (su nombre, en realidad) y el verbo «poner» cuando pretendíamos que representasen la cantidad, ya que no existe en nuestra lengua un verbo diferenciado para escribir cifras o cualquier otra notación de cantidad.

$\mathrm{El}$ análisis de las producciones nos permitió:

a) Comprobar si los elementos y compuestos eran distintos, y cómo se relacionaban con las regularidades gráficas de los sistemas convencionales.

b) Evaluar si utilizaban la escritura para anotar palabras (en este caso el nombre de los objetos) y cifras para cantidades (en este caso la cantidad de objetos). Es decir, evaluar la adecuación de cada sistema a su función comunicativa representacional.

c) Explorar la relación entre el conocimiento formal y la adecuación de los sistemas a su función comunicativa representacional.

\section{METODO}

Sujetos: Un total de 56 niños divididos en tres grupos de edad tomaron parte en este estudio; 20 estaban en primero de parvulario (edad media 4,4), 20 en 
segundo de parvulario (edad media 5,6) y 16 en el primer curso de la escuela primaria (edad media 6,6). Los sujetos fueron escogidos al azar de la lista de niños que asistían a esas clases en una escuela pública de un barrio de Barcelona de la zona norte. La lengua de la escuela era el catalán, pero casi el $60 \%$ de los niños que concurrían a las clases que participaron en el estudio hablaban castellano en sus hogares. Sólo los niños que fueron entrevistados en castellano o catalán - según su lengua de origen - se incluyen en este trabajo. La enseñanza sistemática de la lectura comenzaba en esa escuela en primer grado, pero los niños realizaban actividades de copia de palabras y frases, de reconocimiento de letras y cifras, desde el parvulario. Se trataba, por tanto, de niños «notacionalmente» estimulados. Para ser incluido en la muestra el niño o niña debía saber hacer algunas letras, cifras y escribir su nombre. Para comprobar esto les solicitamos que hicieran algunas letras y números, los que supieran hacer. A veces hicieron letras, cuando les solicitamos «hacer números» y viceversa, no conocían la referencia específica del término metalingüístico que empleamos. Pero lo importante para nuestros propósitos es que produjeron elementos de cada uno de los sistemas para cada solicitud y ninguno hizo un dibujo. Esto significa que en lo que respecta a la denominación genérica y a algunos elementos convencionales los niños diferenciaban entre ambos tipos de notaciones y no las confundían con el dibujo.

Materiales: Utilizamos cuatro pares de figuras de cartón de $4 \mathrm{~cm}^{\star} 6 \mathrm{~cm}$, sobres y etiquetas. En tres de los pares estaban dibujados los mimos objetos en diferente cantidad (por ejemplo, dos ruedas en una figura, cinco ruedas en la otra) y en el cuarto par aparecían diferentes objetos en igual cantidad (tres guardias en una figura y tres camiones en la otra). Los nombres de los objetos elegidos variaban en número de sílabas: desde bisilábicos (coche) a tetrasilábicos (semáforo). Las cardinalidades incluían 1, 2, 3, 5 y 28.

Procedimiento: Mostrábamos a cada niño/a una tarjeta y le pedíamos que indicara qué había dibujado en ella, inmediatamente le pedíamos que escribieran para saber qué bay (repitiendo lo que había dicho que hubiera dibujado). Luego le mostrábamos la otra tarjeta correspondiente al par - repitiendo el mismo proceso-. Cada vez que el niño terminaba de escribir le pedíamos que leyera lo escrito. Inmediatamente después le volvíamos a señalar la tarjeta preguntándole cuántos había (repitiendo lo que habían dicho que hubiera dibujado). Luego le mostramos la otra tarjeta correspondiente al par $-\mathrm{y}$ así sucesivamente-. El orden de presentación de las preguntas fue contrabalanceado. Este diseño nos permitió averiguar si las notaciones diferían, en qué aspectos y si el niño conservaba las mismas inscripciones para los mismos objetos o cantidades y modificaba las inscripciones para diferentes objetos o cantidades.

La hipótesis general fue que las inscripciones serían diferentes y que encontraríamos en ellas muchas de las restricciones de los correspondientes sistemas convencionales.

\section{RESULTADOS}

El análisis de las producciones se realizó en varios pasos atendiendo a las características formales de los sistemas, a la situación de producción y al proceso de producción e interpretación. El primero consistió en comprobar cuántos tipos de inscripciones aparecían en las producciones. Luego analizamos los ele- 
mentos y los compuestos de cada tipo de inscripción. Finalmente, una vez analizados los aspectos formales exploramos la adecuación funcional de las inscripciones. Es decir, cómo eran usadas para informar acerca del contenido de las tarjetas.

Número y tipo de inscripciones

Al clasificar las producciones según el número de tipos de inscripciones diferentes hemos discernido cuatro categorías:

1) producciones en las cuales dos tipos de inscripciones, una de ellas icónica. La inscripción icónica consiste en dibujos esquemáticos de los objetos que hay en las tarjetas, tantos dibujos como objetos. Junto a ellos aparece generalmente una inscripción no icónica, que parece una escritura;

2) producciones en las que apareciera inscripciones del mismo tipo (pero ambas son simil-escrituras o escrituras convencionales). Ningún niño o niña produjo una inscripción que parece una cifra aislada como inscripción de tipo único;

3) producciones en las que aparecen dos inscripciones de tipo diferente, una . escritura o simil-escritura y la otra de una o varias cifras;

4) producciones en las que aparecen tres inscripciones de tipo diferente, una de ellas icónica.

La Figura 1 presenta un ejemplo de cada una de estas categorías. Hemos escogido las producciones que cinco niños diferentes hicieron para la tarjeta en la cual hay dibujadas 5 ruedas.

En el recuadro superior se reproducen los dos tipos de inscripciones, una icónica, la otra símil-escritura. Debajo, a la izquierda, dos inscripciones del mismo tipo - símil-escritura - que otro niño produjo y a la derecha dos del mismo tipo pero de escritura convencional. En tercer lugar, hacia abajo, dos inscripciones de distinto tipo, una símil-cifra y la otra símil-escritura. En la parte inferior tres inscripciones de distinto tipo, dibujo, cifras y escritura, producidas por otro niño.

La evaluación inicial se hizo para cada par de tarjetas por separado, pero, dado que no se encontraron diferencias en la distribución de las categorías por tarjetas, para la categorización final se consideró la categoría más frecuentemente usada por cada niño para todas las tarjetas. Un examen chi cuadrado (4 categorías de tipo de inscripciones por 3 grupos de edad) mostró que no hay diferencias significativas por edad. Sin embargo, se dieron ciertas tendencias que vale la pena comentar. Para ello presentamos la tabla de distribución de frecuencias, por grupo de edad.

\section{TABLA I}

Distribución de producciones según el número de inscripciones diferenciadas por edad*

\begin{tabular}{c|cccc|c} 
Edad & Número de inscripciones diferenciadas & \multicolumn{2}{c}{${\text { Total }{ }^{* *}}^{*}$} \\
\hline & $\begin{array}{l}\text { Dos tipos de ins- } \\
\text { cripciones, una } \\
\text { icónica }\end{array}$ & $\begin{array}{c}\text { Dos inscripciones } \\
\text { del mismo tipo }\end{array}$ & $\begin{array}{l}\text { Dos inscripciones } \\
\text { de tipo diferente }\end{array}$ & $\begin{array}{c}\text { Tres inscripciones } \\
\text { de tipo diferente, } \\
\text { una icónica }\end{array}$ & \\
5 años & 4,7 & 25 & 50 & 5 & 100 \\
6 años & 12,5 & 4,8 & 71,4 & 19 & 100 \\
\hline
\end{tabular}

${ }^{\star} \mathrm{N}=56 \quad{ }^{\star \star}$ en porcentajes 
FIGURA 1

\section{5 ruedas}

Dos tipos de inscripciones una icónica

Dos inscripciones

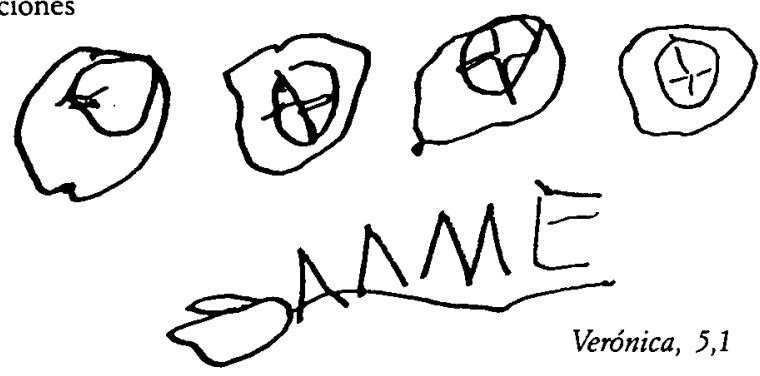

del mismo tipo

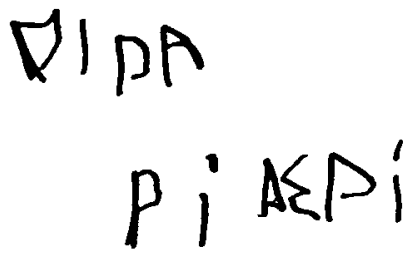

Silvia, 4,8 cincrudas

Alba, 6,8

Dos inscripciones

de tipo diferente

Tres inscripciones de tipo diferente, una icónica<smiles>C1CCCCCC1</smiles>
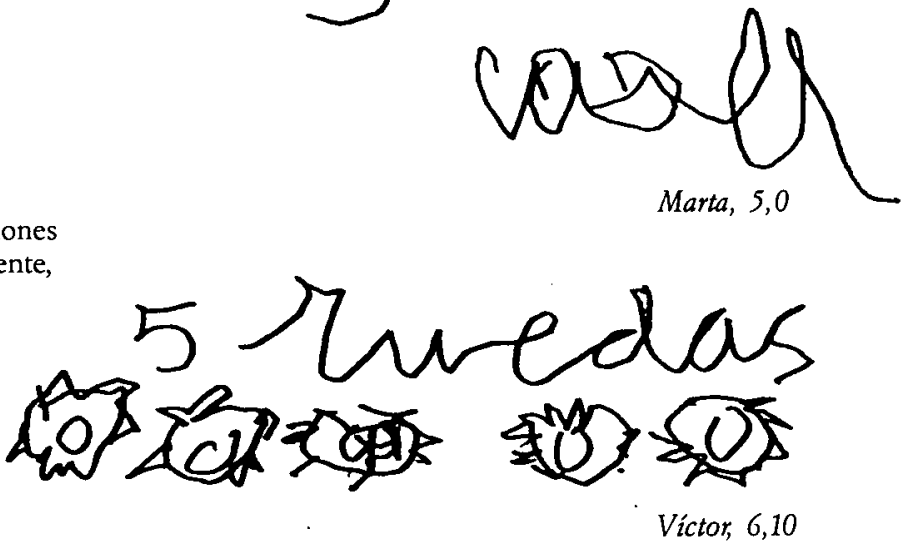

Producciones de seis niños presentando diferente número y tipo de inscripciones

Más del $50 \%$ de los niños en todos los grupos de edad produjo dos inscripciones diferentes, una para número y otra para escritura. Respecto a los niños que produjeron dos inscripciones del mismo tipo - algunos del grupo de 4 años y otros del grupo de 6 años - la escritura es la notación privilegiada, aunque como veremos en los próximos análisis se trata de escrituras de nivel muy diferente. Pensamos que para los niños de 4 años las escrituras notan los nominales y su cantidad como dimensiones indiferenciadas. La manera en que los niños 
interpretaron, en general, sus notaciones parece apoyar esta especulación. Los niños solían decir el sintagma completo «dos ruedas» al señalar lo escrito. Los de 6 años, en cambio, producen el mismo tipo de inscripción porque escribieron convencionalmente tanto el nombre de los objetos como su cantidad con letras y no tenemos muy claro por qué lo hacen.

En las producciones de más del $25 \%$ de los niños en todas las edades aparecieron formas icónicas, pero también éstas parecen cumplir funciones comunicativas diferentes entre los pequeños y los mayores. La forma icónica aparece en los pequeños, otra vez, como la única que refleja la cualidad y la cantidad de objetos. Entre los mayores, parecería ser una forma que agrega información a la provista por la escritura y la cifra. Volveremos sobre esta interpretación al analizar la adecuación funcional de las inscripciones.

\section{La diferenciación formal}

El próposito de este análisis fue averiguar si las inscripciones utilizadas por los niños se diferencian de acuerdo con las retricciones de la escritura y del sistema de notación numérica.

\section{Tipo de elementos y nivel de convencionalidad}

En la sección anterior vimos que ningún niño produjo sólo formas icónicas. Los que las usaron yuxtaponían a ellas alguna inscripción no icónica. En lo que sigue veremos el nivel de convencionalidad de los elementos utilizados por los niños en sus inscripciones no icónicas. Cuando los niños usaban dos tipos de inscripciones y una de ellas era icónica resultaba difícil decidir si ésta correspondía a su representación de escritura o de número. Por eso decidimos considerarla según hubiera sido elicitada por la instrucción «escribe qué hay» o por la instrucción «pon cuántos hay». (Esa es la razón por la cual el total de sujetos en el grupo de 4 años es de 18 en lugar de 20.) Al clasificar las producciones según el nivel de convencionalidad de los elementos hemos discernido cinco categorías:

1) Elementos no icónicos ni convencionales: el niño utiliza palotes, rayas o círculos esquemáticos que no son icónicos pero tampoco se reconocen como elementos convencionales o símiles convencionales.

2) Cadenas simil-escritura: el niño utiliza cadenas de letras cualesquiera o de trazos discontinuos símil-escritura.

3) Elementos únicos símil-cifras

4) Letras convencionales: el niño utiliza letras con valor sonoro convencional

5) Cifras convencionales: el niño utiliza cifras según su valor convencional.

En la Tabla II presentamos la distribución de sujetos por nivel de convencionalidad de los elementos en escritura y notación numérica.

Obviamente el nivel de convencionalidad de los elementos aumentó con la edad de los sujetos. A los 4 años ningún niño utilizó escritura convencional, a los 5 años el $60 \%$ usa letras y a los 6 años lo hace la totalidad de los niños. Este dato es de por sí interesante, ya que para ser incluidos en la muestra los niños debían saber trazar letras; sin embargo, evidentemente no todos los que sabían las usaron cuando debían representar algún contenido. Ningún niño utilizó cifras para escribir, pero varios niños utilizaron escritura convencional o símil-escritura para notar cantidad. En cuanto al uso de las cifras, a pesar de 
que todos sabían «hacer números», sólo el $35 \%$ los usó para saber cuántos bay. Observemos que el $39 \%$ de los niños utilizaron cifras convencionales a los 4 años, indicando un uso más precoz que el de las letras. Estos resultados indican ya que debemos distinguir entre «saber letras» $\mathrm{y}$ «saber usarlas» en una situación comunicativa.

\section{TABLA II}

Distribución de sujetos por tipo de elementos en escritura y número por grupo de edad*

\begin{tabular}{|c|c|c|c|c|c|c|}
\hline Edad & Convencionali & de los elemer & & & & Total $^{\star *}$ \\
\hline & $\begin{array}{l}\text { Escritura con } \\
\text { valor } \\
\text { convencional }\end{array}$ & $\begin{array}{c}\text { Cifras con } \\
\text { valor } \\
\text { convencional }\end{array}$ & Símil escritura & Símil cifra & $\begin{array}{l}\text { Elementos } \\
\text { no icónicos ni } \\
\text { convencionales }\end{array}$ & \\
\hline $\begin{array}{l}4 \text { años } \\
\text { Escr. }\end{array}$ & 0 & 0 & 89 & 0 & 11 & $100(18)$ \\
\hline Núm. & 0 & 39 & 27 & 11 & 22 & $100(18)^{* * *}$ \\
\hline $\begin{array}{l}5 \text { años } \\
\text { Escr. } \\
\text { Núm. }\end{array}$ & $\begin{array}{c}60 \\
0\end{array}$ & $\begin{array}{c}0 \\
19\end{array}$ & $\begin{array}{c}40 \\
0\end{array}$ & $\begin{array}{l}0 \\
0\end{array}$ & $\begin{array}{l}0 \\
1\end{array}$ & $\begin{array}{l}100(20) \\
100(20)\end{array}$ \\
\hline $\begin{array}{l}6 \text { años } \\
\text { Esct. } \\
\text { Núm. }\end{array}$ & $\begin{array}{c}100 \\
19\end{array}$ & $\begin{array}{c}0 \\
81\end{array}$ & $\begin{array}{l}0 \\
0\end{array}$ & $\begin{array}{l}0 \\
0\end{array}$ & $\begin{array}{l}0 \\
0\end{array}$ & $\begin{array}{l}100(16) \\
100(16)\end{array}$ \\
\hline
\end{tabular}

* $\mathrm{N}=56 \quad * \star$ en porcentaje $\quad * \star \star$ Una de las inscripciones era icónica.

Notemos que sólo el $10 \%$ de los niños utilizaron lo que definimos como «elementos no icónicos ni convencionales» (palotes, circulitos, etc.) y lo hicieron más para representar números que para escritura. Es evidente que los niños tendieron a utilizar los elementos gráficos que el medio notacional les ofrece más que a inventarlos. Sin embargo, tal como veremos en el siguiente apartado, los utilizaron de manera algo peculiar, no precisamente según su función comunicativa convencional.

\section{Número de elementos por compuesto:}

Teniendo en cuenta que una de las restricciones diferenciales entre número y escritura atañe al número de elementos generalmente comprendido en un compuesto, nuestro siguiente análisis se centró en esta característica de las producciones. Contamos la cantidad de elementos por compuesto para cada inscripción y definimos el rango por niño y por tipo de inscripción (escritura y número). El rango se define por la diferencia entre la cantidad de elementos que aparecen en la inscripción que contiene menos y en la que contiene más elementos. Se toman las cuatro que produjeron para escribir y las cuatro que produjeron para notar cantidades, separadamente. Al clasificar las producciones discernimos las siguientes categorías:

1) Inscripciones que tienen como mínimo un elemento pero cuyo rango máximo por grupo de inscripcione no es mayor a 1 (es decir, 1 elemento para todas las producciones o entre 1 y 2 elementos).

2) Inscripciones con el mismo rango (no mayor a 1) pero cuyo número mínimo es más de 1 . 
3) Inscirpciones que tienen como mínimo un elemento pero cuyo rango máximo es de 4, por grupo de inscripciones.

4) Inscripciones con el mismo rango (de 1 a 4) pero cuyo mínimo es más de 1. Es decir, no hay ninguna inscripción con un solo elemento.

5) Inscripciones que tienen como mínimo un elemento pero cuyo rango por grupo de inscripciones es mayor a 4 .

6) Inscripciones que incluyen un número de elementos con un rango mayor a 4, pero cuyo mínimo es más que 1 . Es decir, no hay ninguna inscripción con un solo elemento. Las inscripciones en las cuales resultaba imposible contar el número de elementos se incluyeron en esta categoría.

En la Tabla III aparece la distribución de niños por rango de número de elementos y grupo de edad, en escritura y número.

TABLA III

Distribución de sujetos por cantidad de marcas, en escritura y numero, por grupo de edad*

\begin{tabular}{|c|c|c|c|c|c|c|c|c|}
\hline \multirow[t]{2}{*}{ Edad } & & & & & & & \multicolumn{2}{|r|}{ Total $\left.\right|^{* \star}$} \\
\hline & $\begin{array}{l}\text { Rango } \\
\text { máximo 1 } \\
\text { Cantidad } \\
\text { mínima 1 }\end{array}$ & $\begin{array}{l}\text { Rango } \\
\text { máximo } 1 \\
\text { Cantidad } \\
\text { mínima + } 1\end{array}$ & $\begin{array}{l}\text { Rango } \\
\text { máximo } 4 \\
\text { Candidad } \\
\text { mínima } 1\end{array}$ & $\begin{array}{l}\text { Rango } \\
\text { máximo } 4 \\
\text { Cantidad } \\
\text { mínima +1 }\end{array}$ & $\begin{array}{l}\text { Rango } \\
\text { mayor de } 4 \\
\text { Cantidad } \\
\text { mínima } 1\end{array}$ & $\begin{array}{l}\text { Rango } \\
\text { mayor de } 4 \\
\text { Cantidad } \\
\text { mínima }+1\end{array}$ & Incontable & \\
\hline $\begin{array}{l}4 \text { años Esct. } \\
\text { Núm. }\end{array}$ & $\begin{array}{c}0 \\
30\end{array}$ & $\begin{array}{c}10 \\
0\end{array}$ & $\begin{array}{c}0 \\
15\end{array}$ & $\begin{array}{l}55 \\
0,5\end{array}$ & $\begin{array}{l}10 \\
40\end{array}$ & $\begin{array}{c}15 \\
0\end{array}$ & $\begin{array}{l}10 \\
10\end{array}$ & $\begin{array}{c}100(18) \\
100(18)^{*}\end{array}$ \\
\hline $\begin{array}{l}5 \text { años Esct. } \\
\text { Núm. }\end{array}$ & $\begin{array}{c}1 \\
13\end{array}$ & $\begin{array}{l}3 \\
0\end{array}$ & $\begin{array}{l}0 \\
3\end{array}$ & $\begin{array}{l}16 \\
0\end{array}$ & $\begin{array}{l}0 \\
3\end{array}$ & $\begin{array}{l}0 \\
0\end{array}$ & $\begin{array}{l}0 \\
1\end{array}$ & $\begin{array}{l}100(20) \\
100(20)\end{array}$ \\
\hline $\begin{array}{l}6 \text { años Esct. } \\
\text { Núm. }\end{array}$ & $\begin{array}{c}0 \\
14\end{array}$ & $\begin{array}{l}4 \\
0\end{array}$ & $\begin{array}{l}0 \\
0\end{array}$ & $\begin{array}{l}9 \\
0\end{array}$ & $\begin{array}{l}0 \\
0\end{array}$ & $\begin{array}{l}2 \\
2\end{array}$ & $\begin{array}{l}1 \\
0\end{array}$ & $\begin{array}{l}100(16) \\
100(16)\end{array}$ \\
\hline
\end{tabular}

${ }^{*} \mathrm{~N}=56 \quad$ ** en porcentaje

La distribución de sujetos es muy diferente para escritura y número, sobre todo al comparar la cantidad de niños que producen inscripciones con un solo elemento. Sólo dos niños, uno de 4 y otro de 5 años, usaron elementos únicos para escribir los nombres de los objetos, en cambio hay cincuenta y cuatro niños que usaron un solo elemento para notar cardinalidad. La mayoría de los niños produjeron para «escritura» inscripciones con rango de hasta 4, esto es, la diferencia entre la inscripción con menos elementos y con más no fue mayor de 4, y la mínima cantidad de elementos por inscripción fue más de 1. Es decir, ninguna inscripción tuvo un solo elemento. En cambio, para la notación de la cantidad, la mayoría de los niños produjeron inscripciones que incluyeron como mínimo un solo elemento y como máximo dos elementos. Obsérvese que en la tabla aparecen cinco niños en el grupo de 4 años y tres en el grupo de 6 años en la categoría 6, que agrupa aquellos niños que produjeron inscripciones con un mínimo de elementos superior a 1 , pero cuyo rango era mayor a 4 . Los niños de 4 y 6 años están en esta categoría por distintas razones. Los niños de cuatro años produjeron muchos elementos porque su escritura aún no tiene control de cantidad, en cambio. los mayores produjeron muchos elementos porque escribieron la cantidad junto al nombre del objeto (por ejemplo, Beitisietesemaforos). Aunque pocos niños eligieron esta solución notacional nos parece 
interesante referirnos a ella, y a que parece coherente con la interpretación general de los resultados.

\section{Indice de contigüidad:}

Un indicador adicional de la diferenciación entre sistemas apareció al constatar el índice de contigüidad. Este se calculó contando el número de elementos repetidos adyacentes sobre el total de elementos producidos por el niño, separadamente para escritura y notación de cantidad. El índice medio de contigüidad fue de 1.077 (DS 2.78) para escritura y 5.778 (DS 22.44) para cantidad. La diferencia es evidente. Un análisis por grupo de edad manifestó la misma tendencia.

$\mathrm{Al}$ indicar las restricciones propias de cada sistema, señalamos que la repetición es aceptada en las notaciones que se utilizan para cantidad, pero que no es frecuente en la escritura. Los resultados que acabamos de presentar porien en evidencia que esto es también así desde el punto de vista del sujeto que aprende. La diferencia entre los índices de contigüidad, más bajo para escritura y más alto para notación de cantidad, demuestra que la variedad intracompuesto es considerada una restricción en la escritura y no en la notación de la cantidad.

\section{Ligazón entre elementos de un compuesto:}

La ligazón de las inscripciones fue evaluada separadamente para escritura y notación de la cantidad, según dos categorías de respuesta (ligada/no ligada). Un niño era incluido en la categoría «no ligada» cuando por lo menos seis de las ocho inscripciones por dominio aparecían no ligadas, y viceversa.

En la Tabla IV presentamos la distribución de niños según su utilización de inscripciones ligadas o no en escritura y notación numérica.

TABLA IV

Distribución de los sujetos por ligazón de elementos en las inscripciones y grupo de edad, en escritura y número*

\begin{tabular}{c|cc|c|ccc|c} 
Edad & Escritura & \multicolumn{2}{c}{ Total } & Número & Total $^{\star \star}$ \\
\hline & Ligada & No ligada & & Ligada & No ligada & \\
4 años & 50 & 50 & $100(20)$ & 20 & 80 & $100(20)$ \\
5 años & 75 & 25 & $100(20)$ & 0,5 & 95 & $100(20)$ \\
$\mathbf{6}$ años & 93,75 & 6,2 & $100(16)$ & 125 & 87,5 & $100(16)$ \\
\hline
\end{tabular}

$\star N=56 \quad * *$ en porcentaje

Es evidente el predominio de las inscripciones ligadas para escritura y las no ligadas para número. La explicación más simple de este resultado remite a la enseñanza formal. Dado que en la escuela se enseña a escribir comenzando por la letra cursiva mientras que las cifras siempre se enseñan separadas, este resultado indicaría que los niños simplemente reflejaron lo que se les había enseñado. Una explicación más sofisticada indicaría que el niño está reflejando una mayor plausibilidad de separar unidades en la notación de la cardinalidad que en la notación de un enunciado lingüístico. Si bien esta explicación es muy especulativa, nos atrevemos a formularla porque se relaciona con los resultados 
que presentamos respecto a la mayor frecuencia de elementos repetidos en los compuestos numéricos. Parecería que el niño atribuye, y con razón, la separabilidad - manifestada en la notación no ligada - y la iteración — manifestada en la repetición de elementos con mucha más frecuencia a la notación de la cantidad que a la notación de un enunciado lingüístico.

\section{La adecuación funcional}

Una de las distinciones fundamentales que hemos hecho es la de lo notacional como dominio de conocimiento y lo notacional como instrumento de representación y comunicación. Según esta distinción era razonable suponer que la clara diferenciación entre sistemas que apareció cuando se analizaron las notaciones según sus restricciones formales (nivel de convencionalidad de los elementos, número, repetición, ligazón) no se manifestara necesariamente al analizar una notación según su función informativa. El siguiente análisis estuvo encaminado a verificar esta suposición. Teniendo en cuenta qué es lo que los niños debían informar o comunicar (qué hay y cuántos hay en las tarjetas) vimos qué es lo que comunicaron y por qué medios notacionales lo hicieron. Mientras que en el análisis anterior nuestro marco de referencia fue el de las restricciones propias de cada sistema notacional, en el presente análisis la referencia era lo que el niño tenía que comunicar. Los niños deberían dar información sobre las siguientes cuestiones:

a) Qué objetos había en las tarjetas: información cualitativa.

b) Qué los mismos objetos se repetían en tres de los pares de tarjetas y diferían de par en par: información de las similitudes y diferencias cualitativas.

c) Qué la cantidad de objetos variaba entre tarjetas en cada par y entre pares de tarjetas: información de similitudes y diferencias cuantitativas.

Para interpretar las producciones de los niños tuvimos en cuenta cuáles de los aspectos mencionados ( $\mathrm{a}, \mathrm{b}$ y $\mathrm{c}$ ) se reconocían en las producciones. Siguiendo este criterio discriminamos 5 categorías:

1) Diferencias y similitudes por medios icónicos

Producciones en las cuales se refleja tanto la cualidad como la cantidad de objetos debido al uso de formas icónicas (véase figura 1, recuadro superior). La interpretación que los niños hicieron indica que se apoyan en el dibujo como transmisor del contenido y no en la inscripción que lo acompañaba. Solían señalar el dibujo diciendo, por ejemplo: «aquí pone que hay cinco ruedas». Lo que parece escrito, en cambio, no lo interpretaban o decían que lo habian puesto «para escribin» o para «poner el nombre».

2) Diferencias de cantidad por medios no icónicos

Producciones que reflejan las diferencias de cantidad pero no las semejanzas ni las diferencias entre los objetos. Se trata de niños que aumentaban el número de letras al pasar de escribir tarjetas con pocos objetos a tarjetas con más objetos y no modificaban el número de letras cuando la cantidad de objetos era la misma. Es decir, usaban más elementos para escribir (4) coches que para (1) coche, para (5) ruedas que para (2) ruedas, y el mismo número de elementos para escribir policía (3) y camión (3). Sus notaciones no reflejaban la cualidad de objetos porque utilizaban las mismas letras o el mismo patrón símil-escritura en todas sus producciones, independientemente de que los objetos fueran iguales o diferentes. Ahora bien, hubo niños que aumentaron el número de elementos tanto en la escritura como en la notación de cantidad. Otros niños, en cam- 
bio, lo hicieron sólo en la escritura. Asombrosamente, ningún niño utilizó sólo la inscripción-número para notar cantidad. Se dio, sin embargo, una diferencia importante en la manera en que se informó la diferencia de cantidad en la escritura o en la inscripción-número. En la escritura, el niño aumentó letras o multiplicó cadenas escritas, en la inscripción para cantidad el niño repitió tantas cifras como objetos debían ser notados. Es decir, aunque utilizaron la escritura, para una función que no le es propia, respetaron cierta restricción notacional, no repitieron elementos. Siguiendo este criterio - si las diferencias se reflejaron en uno o en los dos tipos de notaciones- hemos dividido esta categoría en dos subcategorías. En la subcategoría (a) se incluyeron los niños que utilizaron la escritura y la inscripción-número. En la subcategoría (b) incluimos los que usaron sólo la inscripción-escritura.

En la siguiente figura aparecen tres producciones de un niño que fue incluido en la subcategoría (a) (parte superior de la figura) y tres de otro niño incluido en la subcategoría (b) (parte inferior de la figura).

\section{FIGURA 2}
(a) 2 ruedas

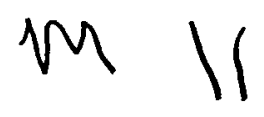
5 ruedas

$$
\text { MNM } 111
$$

2 coches

$$
M
$$

(b) 2 ruedas

$$
\text { ixp ixp }
$$

5 ruedas

$$
\sin (x)|x| x \mid
$$

2 coches

$$
\text { ix ixi }
$$

Miguel Angel, 5,3 
a) Obsérvese que tanto la escritura como la notación para número muestran las diferencias de cantidad. Sin embargo, en escritura el niño agrega elementos mientras que en número el niño repite elementos. Nótese además que las mismas letras fueron utilizadas tanto para palabras similares (ruedas-ruedas) como para palabras diferentes (coche). La notación refleja las diferencias de cantidad peru no las similitudes o diferencias de cualidad. A pesar de que notaciones de diferentes sistemas fueron usadas para diferencias de cantidad, esto se hizo según las restricciones propias de cada sistema - agregado de elementos diferentes en escritura y repetición de elementos, en número-. Parece ser que además de la cantidad el niño intentaba explicitar la recursividad implícita en la noción de cantidad.

b) Obsérvese que sólo la inscripción-escritura muestra las diferencias de cantidad y lo hace por repetición de cadenas y no por repetición o aumento de elementos. Tampoco en este caso se reflejan las similitudes o diferencias entre los nombres de los objetos.

\section{3) Ni diferencias ni similitudes}

Incluye a los niños que no reflejan en sus inscripciones ni las diferencias de cantidad ni las similitudes o diferencias de cualidad.

2 ruedas

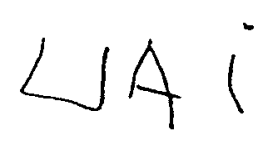

5 ruedas

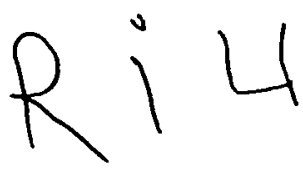

2 coches
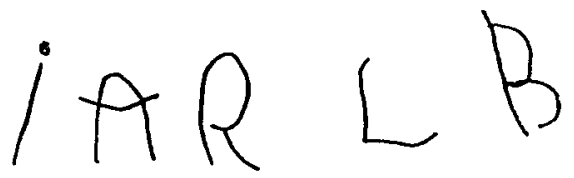

Laura, $4 ; 9$

Producciones de una niña, que no reflejan ni diferencias ni similitudes

Como se puede observar en la Figura 3, estos niños no modificaron el número de letras al pasar de una a otra tarjeta, en cada par, ni tampoco al pasar de uno a otro par de tarjetas. A cada nombre de objeto y a cada cantidad le 
39

corresponde una forma o una combinación de formas diferentes. Estos niños produjeron inscripciones diferenciadas pero en las cuales no se reflejaban ni las diferencias ni las similitudes.

4) Diferencias de cantidad y similitudes por medios no icónicos

Incluye a los niños que reflejan en sus inscripciones la cualidad y la cantidad de lo dibujado en las tarjetas, pero no icónicamente sino por medio de las restricciones propias de cada sistema. Los niños variaron las letras incluidas en sus inscripciones para los nombres de los objetos distintos y las repitieron para los pares similares.

FigURA 4

2 ruedas

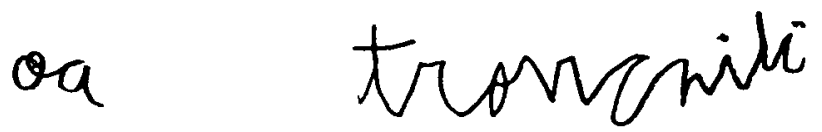

22

5 ruedas

on

al

oa a

3 policías

- $\varepsilon \varepsilon \varepsilon$
55555

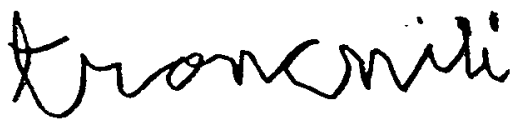

ara

oila

oira

fancic 333

3 camiones

$\varepsilon \varepsilon \varepsilon$

pondre

aiae

a we

333

Khalid, 6; 3

Davinia, 5; 9

Producciones de dos niños, que reflejan diferencias y similitudes por medios no icónicos 
Tal y como se puede observar en la Figura 4 muchos de estos niños ya buscaban correspondencias fonéticas entre las letras y los segmentos ConsonanteVocal de los nominales. La unidad de correspondencia es el segmento silábico, pero el niño lo indica escribiendo sólo la vocal de cada segmento. En lugar de escribir policía escribió oiaa. Sin embargo, a pesar de considerar el patrón fonológico y de utilizar convencionalmente las cifras, siguieron reflejando la diferencia de cantidad, la recursividad y la correspondencia término a término a través de la escritura o a través de la inscripción-número. Esto se logró repitiendo cadenas para corresponder con el número de objetos (Producciones de Khalid, en la parte izquierda de la figura) o repitiendo tantas cifras iguales como objetos debían ser representados (producciones de Davinia en la parte derecha de la figura). Ocho niños demostraron una predilección por la escritura para mostrar diferencias de cantidad, incluso cuando ya realizaban correspondencias fonológicas. Parecería que la cifra no explicita suficientemente la dimensión que el niño intentaba mostrar.

5) Inscripciones funcionalmente diferenciadas

Incluye a aquellos niños cuyas inscripciones reflejan la composición fonológica de la palabra y la cardinalidad, sin mostrar las diferencias de cantidad a través de la escritura. Las cifras pasaron a describir cardinalidades conocidas, pero se mantuvo la predilección por la multiplicidad para las cardinalidades desconocidas. Este es el primer grupo en el cual apareció claramente la adecuación funcional de la escritura, el número y el dibujo.

En la Tabla $V$ presentamos la distribución de sujetos según los aspectos que su notación transmite, es decir, según los niveles de adecuación funcional de sus inscripciones.

TABLA V

Distribución de sujetos según las dimensiones que se reflejan en sus notaciones y grupo de edad*

\begin{tabular}{c|ccccc|c} 
Edad & Nivel notacional & & & & Total \\
\hline & Cualidad y cantidad \\
por medios icónicos & $\begin{array}{c}\text { Diferencias de } \\
\text { cantidad }\end{array}$ & Ni cualidad & Cualidad y cantidad & $\begin{array}{c}\text { Composición } \\
\text { por medios no } \\
\text { icónicos }\end{array}$ & $\begin{array}{c}\text { fonológica de las } \\
\text { palabras y de la } \\
\text { cardinalidad }\end{array}$ & \\
4 años & 35 & 50 & 5 & 10 & 0 & 100 \\
5 años & 5,3 & 5,3 & 16 & 42 & 32 & 100 \\
6 años & 0 & 0 & 0 & 0 & 100 & 100 \\
\hline
\end{tabular}

* $\mathrm{N}=56 \quad$ ** en porcentajes

Los grupos de respuestas presentados manifiestan distintas maneras de aprovechar las cualidades formales de los sistemas notacionales. Los resultados obtenidos demuestran que la linearidad, la discrecionalidad, el número típico de letras que los compuestos de escritura tienen y la no repetición excesiva — se convierten muy temprano en parte de la representación de los niños, que como nuestros sujetos, participan de las actividades de una comunidad alfabetizada-. Muy precozmente, además, el niño relaciona los elementos de la escritura con la pauta sonora de los enunciados lingüísticos, tanto para interpretar lo que ha escrito (categoría 2) como para decidir la cantidad y cálidad de las letras (categorías 4 y 
5). Estos niños también interpretan las inscripciones-número con las correspondientes cardinalidades.

Sin embargo, las cualidades formales y la semántica propia de cada sistema parecen no satisfacer suficientemente la función comunicativa y por eso el niño recurre a otro sistema para mostrar más claramente el contenido de las tarjetas. La presión comunicativa lo lleva a buscar conductas alternativas (y no necesariamente a transformar internamente su representación). No confunde entre escritura y número sino que puede explicitar mejor el contenido del mensaje con un sistema que con el otro. Recordemos que una de las suposiciones básicas de este trabajo era que sólo a partir de la explicitación interna de las propiedades formales y semánticas el niño usaría cada sistema en su función específica aunque con ello disminuyera la explicitación gráfica del contenido. La situación experimental que presentamos a continuación fue diseñada para acceder a este proceso de explicitación interna.

\section{Situación Experimental 2}

En la primera situación experimental comprobamos que las producciones para escritura y cantidad se diferenciaban en sus elementos y en sus restricciones sintácticas. A pesar de ello, hemos visto que algunos niños recurrían al dibujo como la forma de representación más explícita y muchos usaban la escritura para reflejar diferencias de cantidad. Es decir, el conocimiento que tenían acerca de las cualidades que diferencian entre sistemas no garantizó su diferenciación a la hora de tener que transmitir un mensaje. Deberemos demostrar a continuación que la adecuación en el uso comunicacional se produce una vez que las propiedades sintácticas y semánticas que aparecen en las producciones se explicitan internamente. (Aclaremos nuevamente que por explícitamente representado no entendemos verbalmente explicitado sino disponible internamente como contenido, que es posible de ser manipulado.) Para ello debemos mostrar que en los niños que no usaban adecuadamente las notaciones los aspectos notacionales que caracterizaban sus notaciones no habían sido aún internamente explicitadas. Con ese propósito y para poder acceder a los aspectos notacionales que se convierten en objeto de explicitación recurrimos a la misma técnica experimental que ya había sido utilizada en el dominio del dibujo (Karmiloff-Smith, 1990) y a la cual nos referimos anteriormente. Pedimos a cada niño que escriba un nombre que no existe, una letra que no existe y un número que no existe. Es decir, le solicitamos que produzca una inscripción que no pertenece al sistema. Supusimos que para poder producir una inscripción que no es el niño debe poder transgredir intencionalmente lo que si $e^{1}$.

Respecto a esta situación nuestros propósitos fueron:

a) Tener acceso a los objetos de la re-descripción interna, a aquellos aspectos de conocimiento que ya podían ser internamente explicitados y, por tanto, internamente manipulados.

b) Verificar la relación entre el uso comunicativo de las notaciones y aquellos aspectos transgredidos, y por tanto, internamente explicitados.

\section{RESULTADOS}

Para cumplir con el primer propósito clasificamos las producciones de ins-cripciones que no existen comparando las inscripciones que el niño habia pro- 
ducido como letra, número y palabra con aquéllas que el niño había producido como letra que no existe, número que no existe y palabra que no existe. Observando las diferencias gráficas y teniendo en cuenta los comentarios orales que producía al escribir llegamos a las siguientes categorías de respuesta:

\section{Sin transgresión:}

Inscripciones que no presentan ninguna diferencia evidente con las inscripciones normales que el niño produjo. Muchos niños, al pedírseles que produjeran una letra, número o nombre que no existe, simplemente produjeron otro ejemplar del mismo tipo. Los niños que decían no poder hacer «algo que no existe» y se negaban a producir otra notación también fueron incluidos en esta categoría.

Pensamos que se trata de niños que conocían el procedimiento para producir inscripciones pero los componentes de este procedimiento - sus aspectos sintácticos o semánticos - no eran aún parte de una representación interna accesible para ser transformada.

\section{Transgresiones de aspectos gráficos o sintácticos:}

Inscripciones que presentan diferencias evidentes respecto a las inscripciones normales que el niño produjo. Las diferencias se dieron en los aspectos gráficos de los elementos o en los aspectos sintácticos de los compuestos. Algunos niños llegaron incluso a producir un ejemplar de otro sistema. Por ejemplo, un dibujo para números que no existen o para letras que no existen. La deformación gráfica se produjo modificando los contornos, duplicando partes o combinando partes de ejemplares normales. Habitualmente los niños comenzaban trazando el ejemplar normal y luego le agregaban o deformaban partes, hasta que conseguían deformarlo. Obviamente, sólo se percibe que es una deformación si se compara lo que el niño propone como ejemplar que no existe con lo que ha propuesto como ejemplar que existe. Lo que el niño hace es una «transformación» en relación a lo que él mismo ha producido. Las transgresiones de las restricciones sintácticas sólo pudieron darse en los compuestos. Por ejemplo, si un niño escribió nombres con elementos diferentes, los nombres que no existen podían tener elementos repetidos. Si palabras normales tenían muchas letras, las palabras que no existen tenían una sola.

3. Transgresiones de la semántica interna: Inscripciones que presentan diferencias respecto a las inscripciones normales pero no en los aspectos gráficos o sintácticos sino en el aspecto semántico de cada sistema. Para nombres que no existen los niños proponen palabras que «no pueden decirse» o palabras «que no son nada». En su elección de nombres que no existen algunos de estos niños reprodujeron cadenas sin sentido el patrón Consonante-Vocal propio del castellano o el catalán y otros incluso transgredieron el patrón fonológico. Como números que no existen los niños propusieron algunos «tan grandes» que no pueden existir. Números «grandes» son números que tienen muchas cifras, o muchas cifras con ceros intercalados. Parecería que la misma solicitud que fue interpretada por los niños incluidos en la categoría anterior como remitiendo a una modificación de lo escrito, por éstos es interpretada como remitiendo a la correspondencia fonológica o a las relaciones de referencia. La correspondencia fonológica y la cardinalidad se han explicitado internamente y pueden ser trangredidas.

En las Figuras 5, 6 y 7 presentamos ejemplos de producciones de cada una de las categorías. En la Figura 5 se puede observar que las producciones de 
43

Figura 5

Número

3

Nombre

uer

Figura 6

Letra

$m$

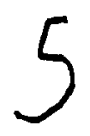

Nombre

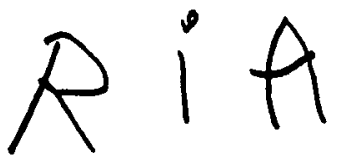

Número
Número que no existe

3

Nombre que no existe

un

Jessica, $4 ; 4$

Producciones de un niño para «números» $y$ «nombres» que no existen - Sin Transgresión

Sergio 5; 6

Nombre que no existe

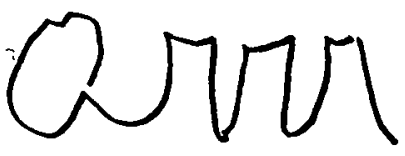

Laura, 4; 9

Producciones de diferentes niños para letras, «números» $y$ «nombres» que no existen: Transgresión gráfica y Transgresión sintáctica 
Jessica para números y nombres que existen y para los que no existen no difieren entre sí. En la Figura 6 Sergio produce una letra que no existe y un número que no existe por una modificación gráfica y una combinación de partes, y Laura transgrede no sólo la variedad interna del compuesto, repitiendo la misma letra tres veces, sino que cambia el tipo de letra. Para poder llegar a tener tantas repeticiones, Laura realizó cuatro intentos sucesivos hasta que finalmente se convencio de que «ése no vale porque tiene dos iguales».

En la Figura 7 aparecen ejemplos de transgresión semántica. Un número con muchos ceros intermedios y una palabra que incluye cuatro consonantes sucesivas, escritas por un niño que ya escribe alfabéticamente.

FiguRA 7

Número

$3^{1}$

Nombre
Número que no existe

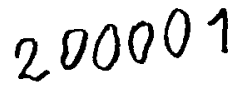

Joan, $6 ; 5$

Nombre que no existe

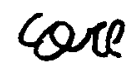

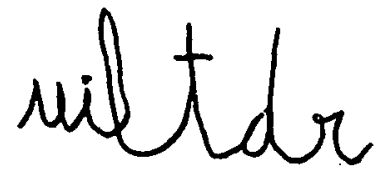

Joan, 6;5

Producciones de un niño para «números» y «nombres» que no existen: Transgresión semántica

Hemos hipotetizado que la adecuación en el uso comunicativo se produciría una vez que las restricciones sintácticas y semánticas se explicitaran internamente. El indicador que usamos para evaluar si esta explicitación se había producido era el tipo de transgresión al producir inscripciones que no existen. Nuestra hipótesis se sostiene si sólo los niños que pueden transgredir aspectos sintácticos o semánticos propios de cada sistema utilizan las cifras y la escritura de acuerdo con sus funciones específicas. Para verificarlo clasificamos los niños según el tipo de transgresión (las tres categorías mencionadas) y según la adecuación funcional. Para la adecuación funcional agrupamos todas las categorías sin adecuación funcional (categorías 1, 2, 3 y 4), separándolas de la categoría con adecuación funcional (categoría 5). El nivel de adecuación funcional se definió una vez para cada niño de acuerdo con la dimensión que reflejaban sus inscripciones en letras, número y nombres que no existen. En las Tabla VI, VII y VIII presentamos el número de sujetos según su nivel de adecuación funcional y el tipo de transgresión realizada, para letras, números y nombres. 


\section{TABLA VI}

Distribución de sujetos por adecuación funcional y aspectos transgredidos en la producción de letras que no existen ${ }^{\star}$

Aspectos transgredidos

\begin{tabular}{lcccc}
\hline $\begin{array}{l}\text { Adecuación } \\
\text { funcional }\end{array}$ & $\begin{array}{l}\text { Sin } \\
\text { transgresión }\end{array}$ & $\begin{array}{l}\text { Transgresión } \\
\text { gráfica }\end{array}$ & $\begin{array}{l}\text { Transgresión } \\
\text { semántica }\end{array}$ & $\begin{array}{l}\text { No } \\
\text { respondieron }\end{array}$ \\
\hline $\begin{array}{l}\text { No diferenciadas } \\
\text { funcionalmente }\end{array}$ & 22 & 38 & 0 & 0 \\
$\begin{array}{l}\text { Diferenciadas } \\
\text { funcionalmente }\end{array}$ & 0 & 25 & 13 & 1,7 \\
\hline
\end{tabular}

${ }^{\star} N=56$ (en porcentajes)

\section{TABLA VII}

Distribución de sujetos por adecuación funcional y aspectos transgredidos en la producción de nombres que no existen*

Aspectos transgredidos

\begin{tabular}{lcccc}
\hline $\begin{array}{l}\text { Adecuación } \\
\text { funcional }\end{array}$ & $\begin{array}{l}\text { Sin } \\
\text { transgresión }\end{array}$ & $\begin{array}{l}\text { Transgresión } \\
\text { gráfica }\end{array}$ & $\begin{array}{l}\text { Transgresión } \\
\text { semántica }\end{array}$ & $\begin{array}{l}\text { No } \\
\text { respondieron }\end{array}$ \\
\hline $\begin{array}{l}\text { No diferenciadas } \\
\text { funcionalmente }\end{array}$ & 22 & 31 & 0 & 0 \\
$\begin{array}{l}\text { Diferenciadas } \\
\text { funcionalmente }\end{array}$ & 0 & 3,5 & 40 & 3,5 \\
\hline
\end{tabular}

$\star \mathrm{N}=56$ (en porcentajes)

\section{TABLA VIII}

Distribución de sujetos por adecuación funcional y aspectos transgredidos en la producción de números que no existen ${ }^{\star}$

Aspectos transgredidos

\begin{tabular}{lcccc}
\hline $\begin{array}{l}\text { Adecuación } \\
\text { funcional }\end{array}$ & $\begin{array}{l}\text { Sin } \\
\text { transgresión }\end{array}$ & $\begin{array}{l}\text { Transgresión } \\
\text { gráfica }\end{array}$ & $\begin{array}{l}\text { Transgresión } \\
\text { semántica }\end{array}$ & $\begin{array}{l}\text { No } \\
\text { respondieron }\end{array}$ \\
\hline $\begin{array}{l}\text { No diferenciadas } \\
\text { funcionalmente }\end{array}$ & 20 & 22 & 11 & 0 \\
$\begin{array}{l}\text { Diferenciadas } \\
\text { funcionalmente }\end{array}$ & 0 & 0 & 40 & 54 \\
\hline
\end{tabular}

$\star N=56$ (en porcentajes) 
Los resultados indican claramente que todos los niños que transgreden la semántica interna del sistema producen inscripciones adecuadas funcionalmente. Este fenómeno se da tanto en escritura como en la notación numérica. Respecto a los aspectos sintácticos, es evidente que su explicitación interna no es suficiente para que el niño pueda producir inscripciones funcionalmente diferenciadas. Muchos niños que usaron los sistemas para funciones que le son ajenas - por ejemplo, la escritura para mostrar diferencias de cantidad - fueron capaces de transgredir las restricciones sintácticas que demostraron en sus producciones.

\section{CONCLUSIONES}

Desde muy temprana edad las restricciones que el niño impone a lo notacional son específicas para cada dominio. En otras palabras, los niños no trataron lo notacional como un área general, sino que cada una de sus realizaciones, dibujo, escritura, notación numérica, fueron exploradas según sus propias restricciones.

Esta diferenciación se dio a varios niveles. Los niños produjeron formas gráficas distintas para dibujar, hacer números o letras. Estos resultados confirman trabajos anteriores con niños hebreoparlantes según los cuales a los 4 años ya se puede distinguir dibujo de escritura en las producciones infantiles (Tolchinsky, Landsmann y Levin, 1985). Parece además que antes de que se haga evidente la diferenciación en los productos gráficos existe una diferenciación en los procesos mismos de producción. Se ha observado que los movimientos que realizan los niños menores de cuatro años al dibujar son distintos de aquellos que realizan al escribir, aunque el producto gráfico resulte parecido (Karmiloff-Smith, 1990).

La diferenciación entre sistemas se dio en las formas gráficas y en los compuestos. La ligazón entre elementos, su número y su variedad interna distinguieron las inscripciones-escritura de las inscripciones-número en la gran mayoría de las producciones observadas. Suponemos que este saber acerca de los notacional se ha construido por una exploración activa de las condiciones formales de los sistemas.

La diferenciación entre sistemas parece ser un punto de partida del conocimiento acerca de lo notacional. Sin embargo, insistiremos una vez más en la distinción que hemos hecho entre el saber notacional, construido por interacción con el medio, y aquel que se pone en juego cuando hay que producir una notación para transmitir un mensaje, en este caso para mostrar qué bay y cuántos hay. A pesar de la precocidad del conocimiento de las cualidades formales, las funciones instrumentales de cada sistema tardan en ser resueltas. Así, algunos niños recurrieron al dibujo para mostrar mejor el contenido de las tarjetas y muchos miños atribuyeron a la escritura la función de mostrar las diferencias de cantidad de los objetos representados en la ellas. Hemos interpretado estos datos no en términos de «confusión» entre sistemas sino en términos de aprovechamiento de cualidades formales conocidas para satisfacer funciones representacionales poco claras. No se trata de que un medio notacional sea más ventajoso que otro, por ejemplo, la escritura más ventajosa que las cifras para describir diferencias de cantidad, sino que el niño aprovechará aquellas características del medio notacional que mejor satisfagan sus propósitos comunicacionales. Es posible que pueda hacer esto porque aún no tiene absolutamente definidas las funciones específicas de cada sistema. 
Respecto a la segunda pregunta que orientó nuestro trabajo acerca de si los niños reconocen las diferencias formales entre los sistemas o sólo sus diferencias funcionales, podemos afirmar lo siguiente: el niño conoce las diferencias formales, pero durante cierto tiempo las características formales de un sistema podrán aprovecharse para funciones que no le son propias. Esta afirmación nos lleva necesariamente a la precedencia de lo formal sobre lo funcional, que era la tercera de nuestras preguntas iniciales. Nuestros datos muestran claramente que el niño sabe acerca de los sistemas y acerca de sus cualidades formales aun antes de haber aprendido a usarlos.

Finalmente, supusimos que para pasar de un conocimiento formal a una adecuación funcional los niños deberían ser capaces de explicitar internamente los aspectos formales que conocían implícitamente. La explicación interna implica transformar estos aspectos en objeto de conocimiento para el sistema cognitivo permitiendo que puedan ser manipulados, y aun transgredidos. La posibilidad de transgredir intencionalmente las restricciones sintácticas y semánticas fue considerada como índice de que éstas habían sido explicitadas internamente. Hemos mostrado cómo los distintos aspectos gráficos y sintácticos podían, con la edad, ser accedidos y transgredidos. Sólo en el grupo de niños que había explicitado internamente la semántica propia de cada sistema - fonología para la escritura y cardinalidad para número- encontramos una utilización de cada sistema según sus funciones específicas. Es decir, para pasar de adherir a las restricciones formales implícitas de cada sistema a usar las notaciones para representar y comunicar contenidos específicos, se requiere la representación explícita de este conocimiento implícito. Al abordar el trabajo, y basándonos en estudios anteriores, supusimos que al conocimiento implícito de las restricciones sintácticas precedería el conocimiento de la semántica interna de los sistemas, pero no teníamos claro si ambos aspectos debían ser internamente explicitados para que el niño pudiera adecuar funcionalmente sus inscripciones. Nuestros resultados demuestran que la explicitación interna de las regularidades sintácticas no alcanza para que el niño llegue a la diferenciación funcional entre escritura y número. Es necesario que explicite internamente la semántica propia de cada sistema. Sólo cuando lo tenga internamente explicitado podrá obviar su explicitación externa. Aclaremos este punto recordando algunos de los ejemplos de utilización de la escritura y el número para mostrar diferencias de cantidad (Figura 3). En ellos aparecen gráficamente manifiestas la iteración y la correspondencia término a término, ambas características de la semántica interna del sistema numérico. Nuestra idea es que aparecen externamente (en forma gráfica) porque aún no se han redescritos internamente, y que una vez esto suceda, el niño podrá tolerar la falta de explicitación (gráfica) de estos aspectos semánticos en el sistema convencional de numeración.

Nuestros resultados sugieren que además de los procesos específicos por dominio notacional, existen algunos procesos generales a todos los dominios. La posibilidad de pasar de explorar la gramática interna de cada sistema a explorar su semántica interna y a partir de su explicitación interna adecuarse a las funciones representacionales y comunicativas propias de cada sistema se da a la misma edad para los dos dominios. Pero además de estos procesos generales en el área de lo notacional, hemos propuesto el proceso recursivo de redescripción (RR) para explicar algunos de los cambios representacionales en este dominio específico. Postular este proceso como característico del desarrollo nos permitió 
formular hipótesis específicas en el dominio notacional tal y como fue posible hacerlo en el dominio del dibujo y el lenguaje. Es probable que trabajando con esta hipótesis podamos descubrir las relaciones de filiación entre los distintos aspectos del conocimiento notacional. El haber descubierto transiciones simultáneas en diferentes dominios y ciertos mecanismos a través de dominios, incluso dominios no notacionales, indicaría que existe un interjuego muy sutil entre procesos cognitivos generales que atañen a lo notacional como dominio general y las restricciones de cada sistema en particular. Es decir, cabría replantearse tanto las posiciones semióticas cómo las modulares.

Debemos recalcar que los resultados que hemos presentado acerca de la discriminación entre sistemas fueron obtenidos en una situación de producción de notaciones. En una situación de elección entre notaciones es posible encontrar una discriminación aún más precoz, tal como aparece en un estudio preliminar realizado (Tolchinsky Landsmann y Karmiloff-Smith, 1992). Suponemos además que, precediendo a la discriminación entre sistemas, encontraremos una clara distinción entre formas icónicas y no icónicas, aun en bebés. La importancia de estos posibles descubrimientos no radica en demostrar la precocidad de la sensibilidad infantil al medio notacional, aunque ello no deja de ser sorprendente. Su interés va más allá del desarrollo individual y compromete las relaciones entre cognición y cultura. Desde diversas perspectivas socioculturales en el estudio de la mente (Wertsch, 1991) se ha enfatizado el papel de los instrumentos culturales en la formación de los mecanismos y contenidos mentales. Desde nuestros datos, y desde los que en el futuro podamos aportar sobre el desarrollo del conocimiento notacional, podremos ver el papel de la arquitectura mental en las características de los instrumentos culturales, en este caso de las características específicas de la escritura y la notación numérica.

El Homo sapiens es una entidad, no una tendencia (Gould, 1990). La posibilidad de simbolizar gráficamente parece ser una marca distintiva de esta entidad.

El ser humano tendría en común con otras especies el uso de herramientas para resolver los problemas inmediatos que el medio platea, pero la idea de utilizar herramientas para registrar los resultados o para evitar mágicamente nuevos problemas es estrictamente humana. Con la notación externa se superan muchas de las limitaciones biológicas de la mente en cuanto a memoria y comunicación. La notación traduce información a otros contextos y crea nuevos contextos. Al separar el conocimiento del sujeto que conoce (Mac Loughan, 1962) y las fases de codificación y decodificación unidas en un acto de comunicación oral (Cohen, 1983), se vencen algunas barreras de tiempo y espacio, pero se abren nuevos espacios de problemas. Los productos gráficos en sí se convierten en espacio de problema. Las sucesivas generaciones de Homo sapiens no sólo usaron el simbolismo gráfico sino que lo reverenciaron y reflexionaron sobre él, sobre sus propiedades y sus aspectos formales. La exploración de las propiedades formales parece ser el punto de partida del desarrollo notacional a nivel del individuo que aprende y probablemente de la diversificación y sofisticación creciente de los sistemas notacionales.

\section{Notas}

1 Distintos aspectos de este trabajo han sido presentados por cada una de las autoras en la conferencia sobre «Domain Specificity and Cultural Knowledge" University of Michigan, octubre, 1990; en el seminario interno del Departamento de Psicología Evolutiva y de la Educación, Universidad 
de Barcelona, España; en los seminarios internos del MRC Unidad para el Desarrollo Cognitivo, Londres; en el seminario interno del Instituto Municipal de Investigación Psicológica Aplicada a la Educación (IMIPAE) Barcelona, España, marzo 1991 y en la Convención de la Sociedad para el Estudio del Desarrollo SRCD, Seattle abril 1991. Agradecemos al Consejo Británico y al Consejo Médico Británico por financiar dos estadias de la primera autora para discutir el proyecto y realizar los estudios piloto. Agradecemos los comentarios realizados por los alumnos del curso de doctorado del Departamento de Psicología Evolutiva y de la Educacción de la Universidad de Barcelona a una versión previa de este trabajo.

2 Esto no quiere decir que siempre identificamos de qué letra se trata, podemos confundirnos, pero sabemos que no puede tratarse de alguna letra desconocida.

3 Utilizamos el término «nombre» para designar los compuestos porque, tal como hemos comprobado en estudios previos, ese es el término que los niños prefieren para designar palabras escritas (véase al respecto Ferreiro y Teberosky, 1979; Ferreiro y Vernon, 1992). Es importante señalar que en el contexto de este trabajo la utilización de términos metalingüísticos es siempre relativa a lo que el niño haya producido y denominado. Será considerado letra lo que el niño haya producido como resultado de nuestra solicitud de «hacer una letra» y será considerado «letra que no existe» lo que el niño haya producido como resultado de nuestra solicitud de hacer una letra que no existe. La comparación de ambos tipos de producciones es también relativa a cada niño.

\section{Referencias}

Allardice, B. (1977). The development of written representations for some mathematical concepts. Joumal of Children's Mathematical Behavior, 2, 135-148.

Boysen, S., y BernTson, G. (1989). Numerical competence in a chimpanzee (Pan troglodytes). Journal of Comparative Psychology, 103, 23-31.

BRUner, J. (1964). The course of cognitive growth. American Psychologist, 19, 1-19.

COHEn, S. (1983). The development of notational skill in children. Unpublished dissertation. Stanford University.

Coulmas, F. (1989). The Writing Systems of the World. Londres: Basil Blackwell.

Cromer, R. (1988). The cognition hypothesis revised. En F. Kessel (Ed.). The development of Language and language researchers Essays in Honor of Roger Brown. Hillsdale, N. J.: Lawrence Erlbaum Associates.

Curtiss, S., y Yamada, J. (1981). Selectively intact grammatical development in a retarded child. UCLA Working Papers in Cognitive Linguistics, 3, 61-91.

Ellis, A., y Young (1991). Human Cognitive Neuropsychology. Hillsdale, N. J.: Lawrence Erlbaum Associates.

FERREIRo, E., y Sinclair, H. (1971). Temporal relationships in language. Joumal Intermational de Psychologie.

Ferreiro, E., y Teberosky, A. (1979). Los sistemas de escritura en el desarrollo del niño. México: $\mathrm{S}$ XXI.

Ferreiro, E., y Vernon, S. (1992). La distinción palabra/nombre en niños de 4 y 5 años Infancia y Aprendizaje, 58, 15-28.

Fonor, J. (1983). The Modularity of Mind. Cambridge, Mass.: MIT Press/Bradford Books.

Freeman, N. H. (1987). Current problems in the development of representational picture production. Archives de Psychologie, 55, 127-152.

Frith, U. (1986). A developmental framework for development dyslexia. Annals of Dislexia, 36, 69-81.

GARDNER, H. (1974). The shattered mind. Nueva York: Vintage.

Goodman, N. (1968/1976). Los lenguajes del arte. Madrid: Seix Barral.

Ifrah, G. (1981). Histoire universelle des chifres. París: Seghers.

Heizelin, J. DE (1962). Ishango. Scientific American, 206, vol. 6, 105-114.

Hughes, M. (1986). Children and number difficulties in leaming mathematics. Oxford: Basil Blakwell.

HarrIs, R. (1990). The origin of writing. Londres: Duckworth.

HARRIs, R. (1992). Notation and writing: Ponencia presentada en The Primer Workshop sobre Oralidad y Alfabetización: Perspectivas históricas y metodológicas. European Science Foundation. Siena: Italia.

Karmilofy.Smith, A. (1979a). A Functional Approach to Child Language. Cambridge: Cambridge University Press.

Karmiloff-Smith, A. (1984). Children's problem solving. En M. E. Lamb, A. L. Brown y B. Rogoff (eds.). Advances in Developmental Psychology, vol. III, Nueva Jersey, Erlbaum, 39-90.

Karminof. SMrTh, A. (1986a). From metaprocesses to conscious access: evidence from children's metalinguistic and repair data. Cognition, 23, 95-147.

Karmiloff-Smith, A. (1986b). Stage/Structure versus Phase/Process in Modelling Linguistic and Cognitive development. En I. Levin (eds.). Stage and Structure: Reopening the debate. Norwood, N. J.: Ablex. 
Karmiloff-SMith, A. (1990a). Piaget and Chomsky on language acquisition: divorce or marriage? First Language, 10, 255-270.

KARMLOFF. SMITH, A. (1990b). Constraints on representational change: Evidence from children's drawing. Cognition, 34, 57-83.

Karmiloff.Smirh, A. (1990c). The human print-out facility: extending biological constraints by cultural tools or electronic mail is runing evolution. Paper given at the Conference on «Domain Specificity and Cultural Knowledge», University of Michigan October.

Karminof.Smith, A. (1992). Beyond Modularity: A developmental Perspective on Cognitive Science. Cambridge, Mass.: MIT Press/Bradford Books.

Karmiloff-Smith, A. (1992). Autoorganizació y cambio cognitivo. Substratum, 1, 19-44.

Kaput,J. (1987). Representation systems and mathematics. En C. Janvier (Ed.) Problems of Representation in the Teaching and Learning of Mathematics. Hillsdale, Nueva Jersey: Lawrence Erlbaum.

Lerol.Gourhan, A. (1971). Prehistoire de l'art Occidental. París: Edition Lucien Mazenod.

Mac Loughan, M. (1962). The Gutenberg Galaxy. Toronto: University of Toronto Press.

MarschaK, A. (1972). The Roots of Civilization. Nueva York: McGraw-Hill.

Gleitman, L. (1989). The structural sources of verb meaning. Papers and Reports on Cbild Language Development, 28, 1-48.

GeLB, I. (1963). The Study of Writing. Chicago: Chicago University Press.

Gould, S. J. (1991). La vida Maravillosa. Barcelona: Crítica.

Sastre, G., y Moreno, M. (1976). Representation graphique de la quantite. Bulletin de Psychologie de l'Universite de Paris, 30, 366-355.

SinCLAIR, A., y Sinclair, H. (1984). Preschooler interpretation of written numerals. Human Learning, 3, 173-184.

SchmandT:Besserat, D. (1977). An archaic recording system and the origins of writing. SyroMesopotamian Studies, 1/2, 2-32.

SchmandT-Besserat, D. (1978). The earliest precursor of writing. Scientific American, 238: 38-47.

Schmandr:Besserat, D. (1990). Symbols in the prehistoric Middle East: Developmental features preceding written communication. En R. Enos (Ed.). Oral and Written Comunication: Historical approaches. Londres: Sage (16-32).

SPEREER, D. (1984). Anthropology and Psychology: Towards and epidemology of representation. Man, 20, 1-17.

Pетттт, L. (1987). On the autonomy of language and gesture: Evidence from the acquisition of personal pronouns in American Sign Language. Cognition, 1-52.

PIAGET, J. (1946). La formation du symbole chez l'enfant. Neuchatel: Delachaux y Niestle.

PIAGET, J. (1976). La toma de conciencia. Madrid: Morata.

Premack, D. (1986). Gavagai. Londres: Cambridge University Press.

Teberosky, A. (1989). La escritura de textos narrativos. Infancia y Aprendizaje.

Teberosky, A.; Tolchinsky Landsmann, L.; Zelcer, J.; Morais, A., y Rincón, G. Segmentation phonologique et acquisition de l'ecriture: une étude dans deux systemes ortographiques (en prensa). Etudes de Linguistique Applique.

TOLCHINSKY LANDSMANN, L. (1990). Early writing development: evidences from different orthographic systems. En M. Spoelders (ed.). Literacy Acquisitions, Lier: Comunication and Cognition and Van In.

Tolchinsky LandSMann, L., y Levin, I. (1985). Writing in preschoolers: An age related analysis. Journal of Applied Psycholinguistic, 6, 319-339.

Tolchinsky Landsmann, L., y Karmiloff-Smith, A. (1991). Using Children's External Notations To Explore Internal Representational Change. Paper presented at the Symposium: The Emergence of Symbolic Thought. SRCD, Seatle, April 1991.

Tolchinsky Landsmann, L., y Karmiloff.Smith, A. (1992). Cognitive Development, 7, 287-300

Tolchinsky Landsmann, L, y Teberosky, A. (1993). Al pie de la letra. Infancia y Aprendizaje, 59/60. Vygorsky, L. (1978). Mind in Society. Cambridge, Ma.: Harvard University Press.

WerTsCH, J. (1991). Sociocultural setting and the Zone of Proximal Development: The problem of

\section{Extended Summary}

Notational refers to humans' capacity to use tools in order to leave permanent traces of our communicative acts. This capacity has given way to different notational systems: drawing, writing, musical notation, number notation, etc. These systems are not only characterized by their elements (e.g., letters for writing and figures for number notation), or by their combinatorial regularities, but also because they fulfill distinct functions: Writing is used to represent language; figures to represent quantities. In previous work we have shown that very 
young children, before learning to read and write, are sensitive to differences between drawing, writing, and number notation. But this does not imply that they know how to use adequately these systems as instruments for representation and communication. In this study we have tried to have access to intrapsychological processes which we asume facilitate the progress between differentiation and the adequate use of notations. The paper presents the results obtained in two studies with 56 children, ages between $3: 8$ and $6: 5$ years, who took part in two production situations. In the first situation, the children were shown a series of pictures depicting the same objects but in different numbers, or different objects but in the same number. The children were asked to write what and how many objects appeared in the pictures. In the second situation, the children were asked to write letters, numbers, or words that did not exist. The purpose of the first situation was to determine: whether the inscriptions which the children made to represent objects differed from the inscriptions they made to represent their number; whether the inscriptions carried the formal retrictions belonging to writing and number notation; and, whether the use of these inscriptions was the one which corresponded to each system. The purpose of the second situation was to find out if formal restrictions and functional adequacy, which would have been demostrated in the first situation, were implicit in their production procedures or were explicitly represented. Only in this case could the children ignore them in order to produce notations that do not exist. The results show that from a very early age the restrictions imposed by the child to the notational system are specific for each domain. Children did not treat the notational system as one general area, each undertaking picture, writing, number notation - was explored according to its own restrictions. Differentiation between systems appeared in the graphic representations and the composites. Inspite of the precocity of knowledge on formal qualities, the instrumental functions of each system takes time to being solved. Therefore, some children resorted to drawing in order to represent what appeared in the pictures, and they used writing to show the different quantities. To proceed from the formal differentiation between systems to its functional adequacy, it is necessary that knowledge of formal restrictions implicit in production procedures becomes internally explicit, and also that it is possible to transgress it. Our results suggest that there is a very subtle interjudgement between general cognitive processes, which involve the notational system as a general domain, and the restrictions from each particular system. 